Review

\title{
Insight of Autophagy in Spontaneous Miscarriage
}

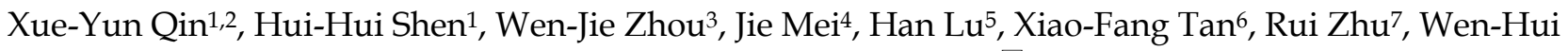 \\ Zhou ${ }^{8}$, Da-Jin Li ${ }^{1}$, Tao Zhang ${ }^{9}$, Jiang-Feng Ye ${ }^{10}$, Ming-Qing Li ${ }^{1,2,11,}$ \\ 1. Laboratory for Reproductive Immunology, Hospital of Obstetrics and Gynecology, Shanghai Medical School, Fudan University, Shanghai 200080, People's \\ Republic of China \\ 2. NHC Key Lab of Reproduction Regulation, Shanghai Institute for Biomedical and Pharmaceutical Technologies, Fudan University, Shanghai 201203, \\ People's Republic of China \\ 3. Center of Reproductive Medicine of Ruijin Hospital, Shanghai Jiao Tong University School of Medicine, Shanghai 200025, People's Republic of China \\ 4. Reproductive Medicine Centre, Department of Obstetrics and Gynecology, Nanjing Drum Tower Hospital, The Affiliated Hospital of Nanjing University \\ Medicine School, Nanjing, 210000, People's Republic of China \\ 5. Departments of Assisted Reproduction, Xin Hua Hospital Affiliated to Shanghai Jiao Tong University School of Medicine, Shanghai 200092, People's \\ Republic of China \\ 6. Reproductive Medicine Centre, Affiliated Maternity and Child Health Care Hospital of Nantong University, Nantong, 226006, People's Republic of China \\ 7. Center for Human Reproduction and Genetics, Affiliated Suzhou Hospital of Nanjing Medical University, Suzhou Municipal Hospital, Gusu School, \\ Nanjing Medical University, Suzhou 215002, People's Republic of China \\ 8. Medicine Centre for Human Reproduction, Beijing Chaoyang Hospital, Capital Medical University, Beijing, 100020, People's Republic of China \\ 9. Assisted Reproductive Technology Unit, Department of Obstetrics and Gynecology, Faculty of Medicine, Chinese University of Hong Kong, Hong Kong, \\ People's Republic of China \\ 10. Division of Obstetrics and Gynecology, KK Women's and Children's Hospital, 229899, Singapore \\ 11. Shanghai Key Laboratory of Female Reproductive Endocrine Related Diseases, Shanghai, 200080, People's Republic of China \\ $\square$ Corresponding author: Ming-Qing Li, E-mail: mqli@fudan.edu.cn
}

(c) The author(s). This is an open access article distributed under the terms of the Creative Commons Attribution License (https://creativecommons.org/licenses/by/4.0/). See http://ivyspring.com/terms for full terms and conditions.

Received: 2021.10.22; Accepted: 2021.12.26; Published: 2022.01.01

\begin{abstract}
In some cases of spontaneous miscarriage (SM), the exact etiology cannot be determined. Autophagy, which is responsible for cellular survival under stress conditions, has also been implicated in many diseases. Recently, it is also surmised to be correlated with SM. However, the detailed mechanism remains elusive. In fact, there are several essential steps during pregnancy establishment and maintenance: trophoblasts invasion, placentation, decidualization, enrichment and infiltration of decidua immune cells (e.g., natural killer, macrophage and $\mathrm{T}$ cells). Accordingly, upstream molecules and downstream effects of autophagy are discussed in these processes, respectively. Of note, autophagy regulates the crosstalk between these cells at the maternal-fetal interface as well. Aberrant autophagy is found in villi, decidual stromal cells, peripheral blood mononuclear cells in SM patients, although the findings are inconsistent among different studies. Furthermore, potential treatments targeting autophagy are included, during which rapamycin and vitamin $\mathrm{D}$ are hot-spots in recent literatures. To conclude, a moderately activated autophagy is deeply involved in pregnancy, suggesting that autophagy should be a regulator and promising target for treating SM.
\end{abstract}

Key words: autophagy, spontaneous miscarriage, trophoblast cells, placentation, decidualization, decidual immune cells

\section{Introduction}

Spontaneous miscarriage (SM) refers to natural pregnancy loss before 20 weeks of gestation, though the exact definition remains controversial. It has drawn great social concern in light of increasing morbidity during latest decades [1]. Main risk factors for SM include fetal chromosomal abnormality [2], maternal infections, uterine structural defects, endocrine disorders, and immune dysregulation [3, 4]. However, in a certain part of cases, especially for recurrent spontaneous miscarriage (RSM), the underlying mechanisms still remain undefined, which urgently deserves more research on etiology, pathogenesis and effective therapeutic methods.

Macroautophagy/autophagy is a self-degradative cellular process in eukaryotic cells, warranting survival under stress circumstances like energy or nutrition shortage, inflammatory events, oxidative stress and endoplasmic reticulum stress [5, 6]. Besides keeping cellular homeostasis, autophagy has been flagged the functionality in physiological and pathological process, for instance, immune response, malignancy, and neurodegeneration [7-9]. Likewise, 
in reproductive system, autophagy has been reported to exist in physiological and pathological endometrium, involving in regulating menstrual cycle, development of endometriosis, adenomyosis and endometrial carcinoma [10-12]. Additional studies have brought further supporting evidence to relationship between autophagy with embryogenesis, implantation, decidualization and pregnancy maintenance [13-15].

In mechanism, autophagy contains five basic steps: nucleation, elongation, maturation, fusion and degradation (Figure 1A). First, autophagy is initiated by isolation membrane namely phagophore which later expands to engulf intracellular cargo and forms autophagosome. After that, autophagosome becomes mature via fusion with lysosome and the cargo is degraded for further recycle finally. In the aspect of molecule, autophagy is activated by the completion of unc-51-like kinase 1 (ULK1) complex, which consists of focal adhesion kinase family interacting protein of $200 \mathrm{kDa}$ (FIP200), Atg13 and ULK1. ULK1 complex is negatively regulated by mammalian target of rapamycin complex 1 (mTORC1) and positively regulated by $5^{\prime}$ adenosine monophosphate-activated protein kinase (AMPK) via directly suppressing and enhancing the activation of ULK, respectively [16]. Followed with phagophore formation, Atg5-Atg12 conjugation interacts with Atg16L to form Atg16L complex. Meanwhile, LC3 precursor convert to LC3-II with the assistance of Atg4B and phosphatidylethanolamine successively [17]. LC3-II locates and inserts into the extending phagophore membrane, typically serving as a critical marker of autophagy activity. Moreover, differing from non-selective autophagy, selective autophagy is regulated by the p62/SQSTM1 paradigm [18]. Considering autophagy is a continuous cellular process, for proper detection, the concept of autophagic flux must be mentioned. To date, both bafilomycin A1, which inhibits lysosomal degradation and chloroquine, which blocks autophagosome-lysosome fusion, are used to evaluate the functional status of autophagic flux [19].

There are several significant events to maintain successful pregnancy: fertilization and embryonic development, trophoblasts invasion, placental development, decidualization, enrichment and infiltration of decidual immune cells (Figure 1B). Recent studies imply that autophagy may associated with SM through regulating decidualization, trophoblast cells and immune cells at maternal-fetal interface $[13,20,21]$. Herein, we discuss the importance of autophagy during early pregnancy and offer a new insight into spontaneous miscarriage and relevant treatment.

\section{Autophagy in fertilization and embryonic development}

Fertilization is the beginning of embryonic development. For mammals, sperm cell penetrates in and donates its DNA to oocyte to form a zygote which will develop into a fetus later. Tentative evidence indicates autophagy might regulate both androcyte and oocyte during fertilization process [22-25]. However, whether and how autophagy affects embryo development via regulating gametes remains unclear. In mouse embryos, autophagy is initiated from fertilization and shortly upregulated during preimplantation period. Zygote derived from knockout Atg5 oocyte and sperm fails to develop beyond the four- and eight-cell stages. But if adopts knockout Atg5 oocyte and wild type sperm, embryonic could continue to develop, indicating maternal Atg5 remained in oocyte cytoplasm might be a protective mechanism [26]. Actually, defective embryo development one of the most common causes for SM [27]. It has now been reported that autophagy trigged by virus disturbs embryo development during early pregnancy $[28,29]$. Besides, obesity could elicit embryonic DNA damage, aberrant histone methylation and impaired autophagy in mice [30]. Survivin, a member of inhibitor of apoptosis protein family which regulates apoptosis and cell cycle, is involved in early embryo development via regulating spindle organization and chromosome alignment. Inhibited survivin leads to accumulated ROS and DNA damage, which induces autophagy and apoptosis in mice embryos [31]. In RSM, an important ROS scavenger, preimplantation factor, is remarkably reduced. Subsequently, accumulated ROS might cause embryo toxicity via autophagy by modulating AMPK/mTOR [32, 33]. These reports indicate that dysregulated autophagy induced by ROS and nuclear stress might impair embryo development, which further triggers pregnancy losses.

\section{Autophagy relates to trophoblasts and placental development}

\section{Autophagy-related genes/molecules in trophoblasts}

Trophoblast cells undertake the tasks of establishing the maternal-fetal interface and can be identified as villus trophoblasts (VTs) and extra-villus trophoblasts (EVTs). VTs are consist of inner layer of cytotrophoblasts (CTBs) and outer layer of syncytiotrophoblasts (STBs), which forms villus structure and enables maternal-fetal energy and nutrients exchange. EVTs mediate invasion, angiogenesis and vascular remodeling to facilitate placentation. 
A

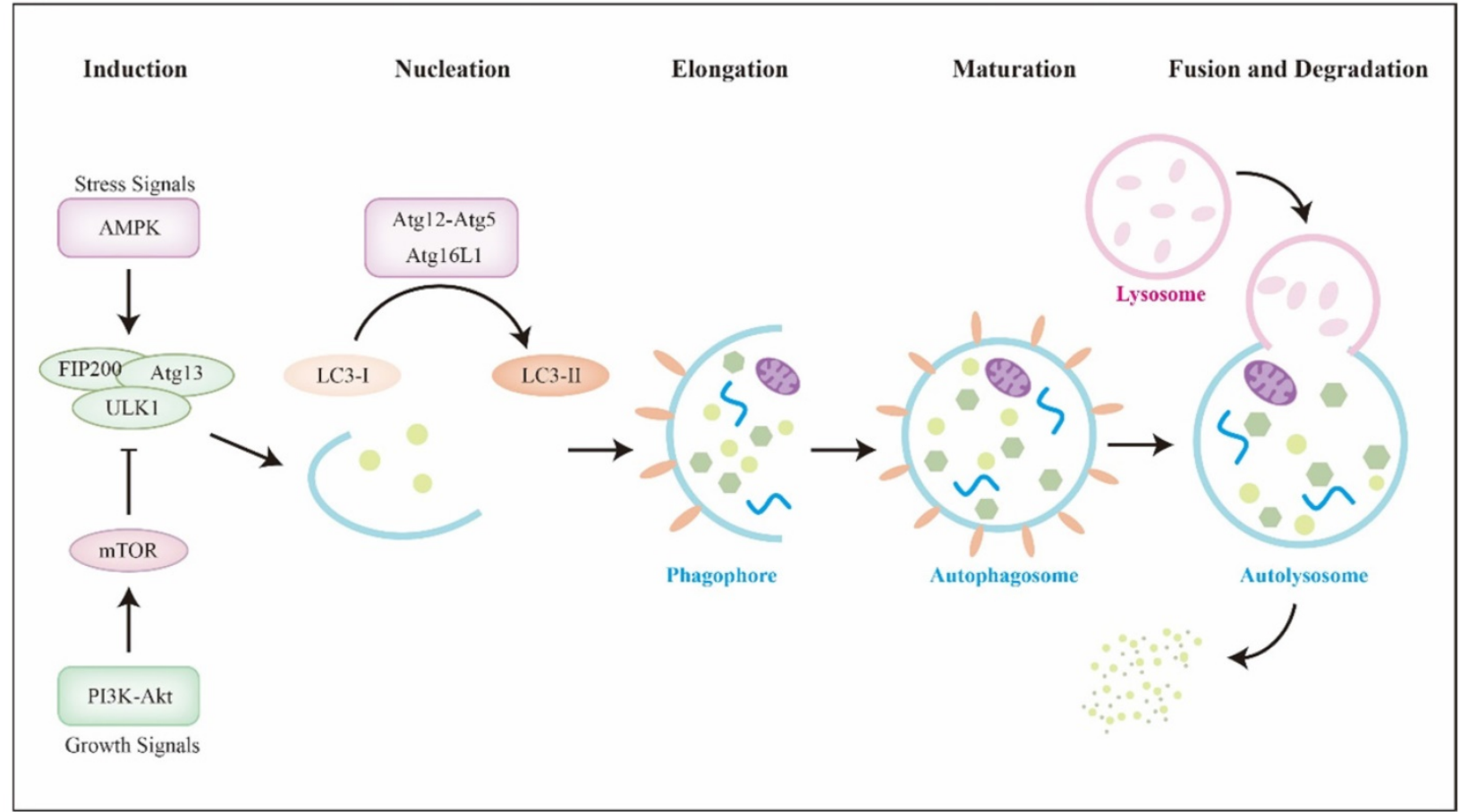

B

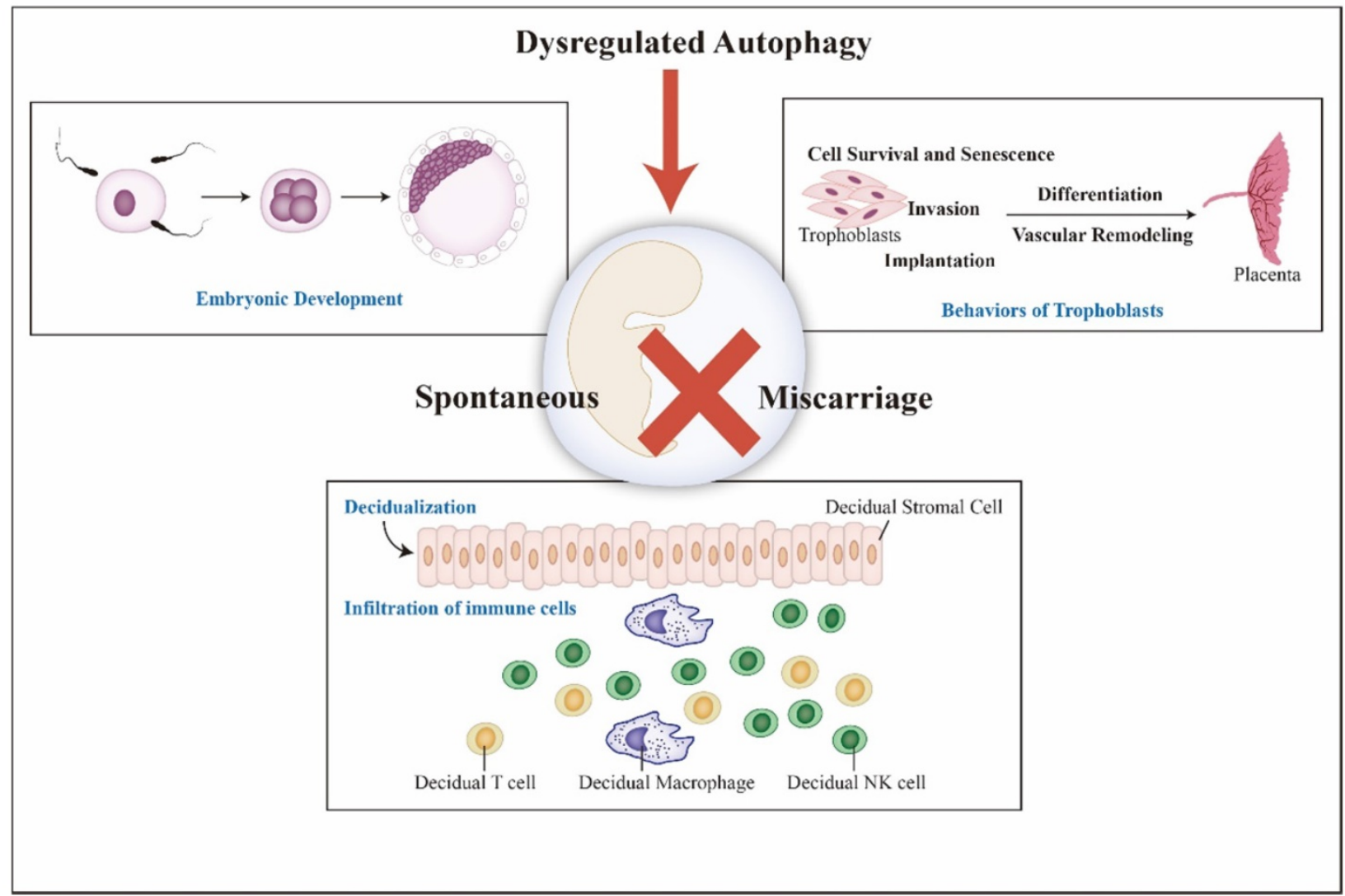

Figure 1. Basic processes of autophagy and key events during pregnancy. A) Induced by stress or growth signals, AMPK and PI3K-Akt are activated. Then, PI3K-Akt upregulates mTOR, an inhibitor of autophagy, to suppress ULK complex (ULK1-FIP200-Atg13). However, AMPK activates ULK complex to initiate autophagy. Autophagy can be divided into five basic steps, including nucleation, elongation, maturation, fusion and degradation. Atg16 complex (Atg5-12, Atg16L) help LC3 locate and insert into extending phagophore membrane. After that, mature autophagosome merges with lysosome and is degraded for cycling. B) Several key events during pregnancy are included. Aberrant autophagy might elicit miscarriage via affecting fertilization and embryonic development, bio-behaviors of trophoblasts, placentation, decidualization and infiltration of immune cells. Abbreviations: AMPK: 5' adenosine monophosphate-activated protein kinase; Atg: autophagy-related genes; LC3: microtubule-associated protein 1 light chain 3; mTOR: mammalian target of rapamycin; PI3K: class I phosphatidylinositol 3-kinase; ULK1: unc-51-like kinase 1 
In normal pregnancy, LC3, a widely used distinguished marker for autophagy, is found to localize in VTs, EVTs and trophoblast-anchoring in term human placentae [34, 35]. Confirmed by electron microscopy, the activity of autophagy predominantly localizes to the STBs with obvious autophagosomes. Meanwhile, other autophagic molecules including Beclin-1, Atg5, Atg9, Atg16L1, p62 and LAMP-2, are all exhibited in VTs [36]. Some upstream regulators of autophagy have been identified to express in human trophoblasts as well. For instance, mTOR mainly expresses in STBs of human placenta and mediates amino acid transport. Fetal growth restriction (FGR) also exhibits mTOR inhibition and increased expression of its inhibitor tuberous sclerosis complex $2[37,38]$. Besides, p53 is located in the first trimester placental CTBs and combats with LC3B-II levels in STBs. Similar results are also observed in BeWo cell line and placental explants [39]. Bcl-2 is another suppressor of autophagy for its efficacy in binding with Beclin-1. In villus explants, Bcl-2 and its family member Bcl-xL are detectable in CTBs but negatively associated with expression of LC3-II [40]. Transcription factor EB (TFEB), a positive regulator for autophagy and lysosomal genes, has been found to express in primary human trophoblasts and EVTs cell lines [41]. However, for specimens taken from placentae of preterm birth patients, autophagy activity is reduced and related markers are differentially distributed. The expression of LC3 and Atg16L1, but not Atg7 or Beclin-1, are higher in STBs than those of CTBs [42]. Compared with health controls, LC3 staining is elevated in STBs of SM patients [35]. These studies display the expression patterns of autophagy-related markers in trophoblasts during normal human pregnancy and their aberrant alterations in pregnancy complications.

Likewise, autophagy-related markers are also exhibited in trophoblast cells of mice. LC3A, B and C mainly concentrate on STBs while giant trophoblast cells express LC3C instead of LC3A [43]. The LC3B staining exists mostly in spongiotrophoblast cells with occasional expression in giant trophoblast cells [44]. Induced autophagy could upregulate LC3B-II and p62 protein in mice placental trophoblasts [45]. Trophoblast-specific conditional Atg7 knockout mice exhibits aberrantly accumulated proteins in placenta probably via reducing TFEB [41], implying an impaired autophagy plays important roles in trophoblast-associated pathological pregnancy conditions, such as preeclampsia (PE). Murine placental Atg4C and Atg7 declines in inflammation-induced preterm labor (IPTL) [46]. IPTL has also been observed in Atg16L1-deficient mice with reduced ability to withstand infection [42].
Overall, these results offer practical clues to autophagy in trophoblast cells. Expression patterns of autophagy-related molecules display in Table 1.

Table 1. Expression of autophagy-related molecules on trophoblasts and decidua

\begin{tabular}{|c|c|c|c|}
\hline Study & Objects & Samples & $\begin{array}{l}\text { Expression of autophagy-related } \\
\text { molecules }\end{array}$ \\
\hline $\begin{array}{l}\text { Avagliano, } \\
2015\end{array}$ & human & $\begin{array}{l}\text { VTs } \\
\text { EVTs }\end{array}$ & LC3, Bcl-2, Bax, HIF-1a \\
\hline Pan, 2018 & human & VTs & LC3 and p62 \\
\hline Curtis, 2013 & human & VTs & $\begin{array}{l}\text { Beclin-1, Atg5, Atg9, Atg16L1, } \\
\text { p62, LAMP-2 }\end{array}$ \\
\hline Roos, 2007 & human & STBs & mTOR \\
\hline Hung, 2017 & human & $\begin{array}{l}\text { term placentae } \\
\text { CTBs }\end{array}$ & mTOR, TSC2, LC3B-II, p62 \\
\hline Gauster, 2018 & human & $\begin{array}{l}\text { BeWo } \\
\text { first trimester } \\
\text { placentae }\end{array}$ & ATGs, p62, LAMP-1, p53 \\
\hline Hung, 2010 & human & CTBs & Bcl-2, Bcl-xL, LC3-II \\
\hline $\begin{array}{l}\text { Nakashima, } \\
2020\end{array}$ & human & $\begin{array}{l}\text { STBs } \\
\text { EVTs }\end{array}$ & $\begin{array}{l}\text { TFEB, LAMP-1, LAMP-2, } \\
\text { cathepsin D }\end{array}$ \\
\hline & mice & placentae & TFEB \\
\hline Cao, 2016 & human & $\begin{array}{l}\text { PTB STBs } \\
\text { PTB CTBs }\end{array}$ & $\begin{array}{l}\text { LC3, Atg16L1 } \\
\text { Atg7, Beclin-1 }\end{array}$ \\
\hline Hiyama, 2015 & mice & $\begin{array}{l}\text { STBs } \\
\text { giant trophoblast cells } \\
\text { spongiotrophoblast } \\
\text { cells }\end{array}$ & $\begin{array}{l}\text { LC3A, LC3B, LC3C } \\
\text { LC3C } \\
\text { LC3B }\end{array}$ \\
\hline Zhu, 2019 & mice & trophoblasts & LC3B-II, p62 \\
\hline Agrawal, 2015 & mice & placentae & $\operatorname{Atg} 4 C, \operatorname{Atg} 7$ \\
\hline Rhee, 2016 & $\begin{array}{l}\text { human } \\
\text { mice }\end{array}$ & $\begin{array}{l}\text { induced DSCs in vitro } \\
\text { decidualizing cells }\end{array}$ & $\begin{array}{l}\text { LC3B } \\
\text { ACC, p-ACC, p-ULK1 }\end{array}$ \\
\hline $\begin{array}{l}\text { Oestreich, } \\
2020\end{array}$ & $\begin{array}{l}\text { human } \\
\text { mice }\end{array}$ & $\begin{array}{l}\text { induced DSCs in vitro } \\
\text { decidualizing cells }\end{array}$ & Atg16L, LC3B-II \\
\hline Su, 2020 & $\begin{array}{l}\text { human } \\
\text { mice }\end{array}$ & decidua & $\begin{array}{l}\text { LC3, p62 } \\
\text { Atg5, LC3, cathepsin B, and p62 }\end{array}$ \\
\hline Lu, 2020 & human & DSCs & LC3-II, p62 \\
\hline Oestreich, & human & induced DSCs in vitro & FIP200 \\
\hline
\end{tabular}

Abbreviations: ACC: acetyl-CoA carboxylase; Atg: autophagy-related genes; CTBs: cytotrophoblasts; DSCs: decidual stromal cells; VTs: villus trophoblasts; EVTs: extra-villus trophoblasts; FIP200: focal adhesion kinase family interacting protein of $200 \mathrm{kDa}$; HIF-1a: hypoxia-inducible factor-1a; LC3: microtubule-associated protein 1 light chain 3; mTOR: mammalian target of rapamycin; STBs:

syncytiotrophoblasts; TSC2: tuberous sclerosis complex 2;PTB: preterm birth;

ULK1: unc-51-like kinase 1; TFEB: transcription factor EB;

\section{Upstream regulators of autophagy for trophoblast cells}

AMPK/mTOR has been identified as upstream regulatory pathway for autophagy. For one thing, AMPK, acting as a cellular energy sensor, is upregulated by energy consumption (elevated ratio of AMP to ATP) [47]. Then, activated AMPK directly phosphorylates ULK [16]. For another, inhibited class I phosphatidylinositol 3-kinase (PI3K) inactivates its downstream Akt and thus represses activity of mTORC1. After that, ULK complex frees from mTORC1 and drifts to an active form [48]. Anyway, activated Atg13 and ULK, aggregates with FIP200 and forms ULK complex in order to initiate autophagy. However, either mTOR or AMPK seems to take an opposite effect on autophagy in trophoblasts. Zhang et al. have reported that moderate inhibition of mTOR 
enhances autophagy while excessive inhibition of mTOR reduces autophagy in trophoblasts. That could be mediated by the functional loop between the O-GlcNAcylation and phosphorylation of Beclin-1 [49]. Additionally, Yang et al. have reported AMPK as a negative regulator of autophagy in primary human trophoblasts [50]. With the treatment of AMPK agonist, fusion and degradation of autolysosomes are impaired. In contrast, administration of AMPK antagonist upregulates LC3B-II and LC3B-II/I ratio with depletion of p62. Simultaneously, impeded mitophagy (decreased PINK1) and mitochondrion fission/fusion (decreased OPA1, MFN1, Drp1, and FIS1) are observed in cocultivation with AMPK antagonist or agonist [50]. On top of different cell lines, whether AMPK antagonist could activate autophagy in an AMPK-independent manner deserves further research.

Inhibited Akt/mTOR promotes autophagy of trophoblast cells. LncRNAH19 targets miR-18a-5p, and the downregulation of H19 promotes autophagy via suppressing PI3K/Akt/mTOR in vitro [51]. Insulin-like growth factor 2 (IGF2) inhibits autophagy as it's an inducer of PI3K/Akt [52]. This effect could be blocked by miR-16-5p but be rescued by ligustrazine [53]. Furthermore, as a downstream pathway of PI3K/Akt, Sonic Hedgehog signaling (Shh) could be inactivated by Akt [54], and thus enhances autophagy with accumulation of autolysosomes [20]. Therefore, Akt/mTOR and Akt/Shh show inhibitory and promotive effects on autophagy respectively, which forms a functional loop.

Status of autophagy changes in response to various outer stress. Since hypoxia regulates CTBs proliferation and invasion to establish maternal-fetal interface during early pregnancy [55], the role of autophagy in this condition should be emphasized. In hypoxia, TFEB is downregulated and thus autophagic flux is disrupted in primary human trophoblasts [41]. Reactive oxygen species (ROS) and endoplasmic reticulum (ER) stress also activate autophagy $[56,57]$ and upregulate LAMP-2 and LC3B-II, which could be confirmed by electron microscopy $[36,58]$. However, discrepancy does exist in status of trophoblastic autophagy under hypoxia [59], which might be explained by different samples, cell lines, severity of hypoxia and interpretation of trophoblast autophagy.

Actually, hypoxia-inducible factor-1a (HIF-1a), a transcription factor that stabilizes in hypoxia, is a critical upstream regulator for autophagy in trophoblasts. Elevated autophagy may act as a compensation for upregulated HIF-1a and aberrant morphological alterations of villus [60]. In physiological hypoxia $\left(8 \% \mathrm{O}_{2}\right)$, autophagy is activated by increased HIF-1a. Whereas, in severe/pathological hypoxia $\left(\begin{array}{lll}2 \% & \mathrm{O}_{2}\end{array}\right)$, persistent overexpression of HIF-1a may impair autophagy in EVTs via soluble endoglin secreted by villus [61]. Besides, hypoxia induces mitochondrial malfunctions and impaired mitophagy of trophoblasts [59, 62]. BNIP3, a mitochondrial protein, competes with Beclin-1 for binding to Bcl-2. Thereby, extricated Beclin-1 triggers autophagy $[63,64]$. BOK and MCL1, both of which are Bcl-2 family members, tightly work as a system to regulate autophagy of trophoblasts in hypoxia. Tilted balance towards BOK induces autophagy which could be rescued by MCL1 [65]. These results suggest that Bcl-2 family members might mediate altered autophagy and dysfunctional mitochondria in hypoxia.

In high glucose condition, SIRT3/AMPK/mTOR activates autophagy and thus inhibits invasion and proliferation of trophoblasts, which could be abrogated through knockdown of Atg5 [66, 67]. Death-associated protein kinase-3 is elevated in placentae of gestational diabetes mellitus, facilitating autophagy flux and autophagosome-lysosome fusion by interacting with synaptosomal-associated protein 29 [68]. With treatment of high glucose, miR-193b is downregulated and elicits insulin-like growth factor-binding protein 5-induced autophagy, which may trigger gestational diabetes mellitus [69]. However, in oxygen and glucose deprivation test, induced placental ER stress inhibits mTOR [38]. Thereby, autophagy is remarkably enhanced with higher level of LC3B-II in primary CTBs, which could be reversed by re-supplementation [58]. These results reveal that trophoblastic autophagy is initiated in both excessive and limited glucose conditions. Upstream regulatory signals of autophagy in trophoblast cells are displayed in Figure 2.

\section{Role of autophagy in regulating biologic behaviors of trophoblast cells}

Trophoblast cell is crucial for embryo implantation and placental development, as well as of great significance for successful pregnancy maintenance, undoubtedly. And autophagy could regulate trophoblast behaviors via different gene expressions or signaling pathways.

\section{Cell survival and senescence}

Autophagy combats with hypoxia-induced apoptosis to facilitate placental trophoblast survival, in which Atg7 is involved in [70], while LC3 and Beclin-1 response differently to sever hypoxia $(<1 \%$ $\mathrm{O}_{2}$ ) [71]. Furthermore, activated autophagy restricts cell apoptosis via degrading caspase-9 in a p62-dependent manner [45]. Sagrillo et al. have 
demonstrated that melatonin protects trophoblast cells from hypoxia via autophagy and Nrf2 pathway [72]. Excessive ROS stimulates NLRP1 inflammasomes and promotes the secretion of IL-1 $\beta$ and CASP1. Meanwhile, both autophagy and apoptosis are increased. When facing ROS, silencing NLRP1 leads to decreased IL-1 $\beta$, CASP1, and NLRP3. Then autophagy will take over to facilitate cell survival via decreasing p62 but elevating LC3-II, Beclin-1, Atg5, and Atg7. On the contrary, inhibited autophagy will elicit the expression of inflammasome [73]. Therefore, hypoxia-induced ROS activates autophagy to ensure trophoblast survival. However, Lee et al. have reported autophagy as an inhibitor of trophoblast viability with accumulation of ROS and ER stress [74]. Cha et al. also points out that TNF-a-induced autophagy mediates intrinsic apoptosis via Atg5 [75], which could be rescued by resveratrol [76]. Though different stress and cell lines they used may account for this discrimination, the possibility that autophagy regulates both adaptive response and damaging process in trophoblasts survival couldn't be ignored. Additionally, $\mathrm{O}_{2}$ concentration may play a predominant role in cellular senescence via autophagy in trophoblast cells. Physiological normoxia $\left(5 \% \mathrm{O}_{2}\right)$ triggers autophagy, resulting in a decrease in senescence phenotypes (decreased SASP,
IL-8, IL-6 secretion). While pathophysiological hypoxia $\left(1 \% \mathrm{O}_{2}\right)$ causes cytotoxicity with the extracellular release of ATP and lactate dehydrogenase. Interestingly, administration of 3-methyladenine (3-MA), an autophagy inhibitor that blocks PI3K, reverses cellular senescence when facing oxygen stress $\left(21 \% \mathrm{O}_{2}\right)$ [77].

\section{Invasion}

For rodents, conditional knockout of Atg7 in placental trophoblasts shows impaired invasion ability with reduced matrix metalloproteinases (MMP) 2 and 9 [78]. However, in human trophoblast cell lines, some studies have regarded autophagy as a negative regulator for trophoblast invasion $[20,51,53$, $79,80]$. According to Zhou et al., aberrantly enhanced autophagy might give rise to inhibited trophoblast invasion in SM patients [81]. Downregulated Beclin-1 partially alleviates invasion inhibition caused by activated autophagy in JAR cell line [20]. In mechanism, autophagy inhibition promotes trophoblast invasion via activating NF- $\mathrm{kB}$ pathway, upregulating MMP-2 and MMP-9 while downregulating TNF-a [80]. TNF-a could also be induced by inflammatory stress, during which activated autophagy might be a protector for trophoblasts via suppressing NF-KB at a cost of

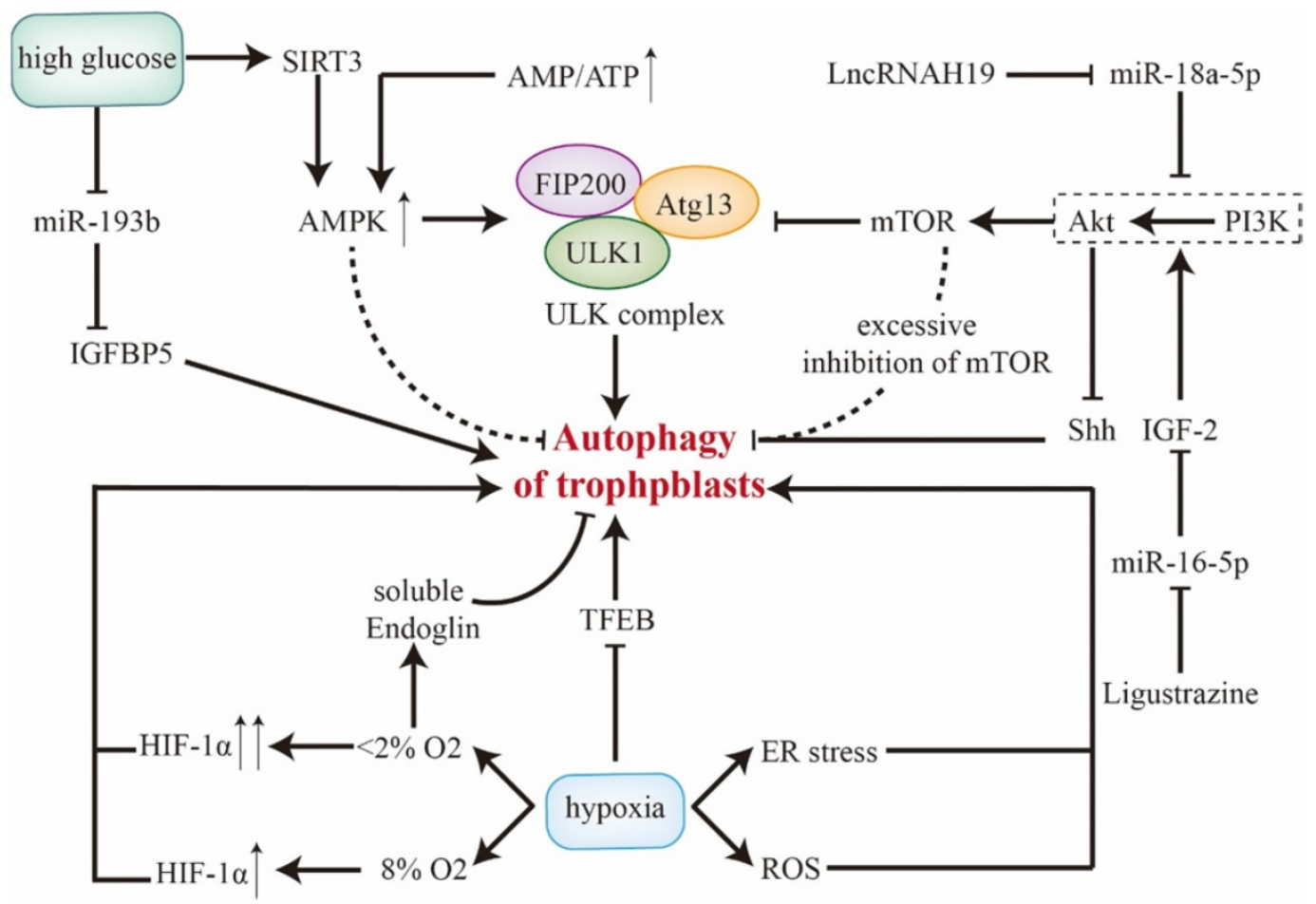

Figure 2. Upstream regulators of autophagy in trophoblast cells. The role of AMPK and PI3K/Akt/mTOR along with related molecules in regulating trophoblastic autophagy are included, during which AMPK and mTOR exhibit dual effects. Besides, SIRT3/AMPK and miR-193b in trophoblasts response for high glucose condition. Of note, different severities of hypoxia modulate autophagy discrepantly. Abbreviations: AMPK: 5' adenosine monophosphate-activated protein kinase; Atg: autophagy-related genes;

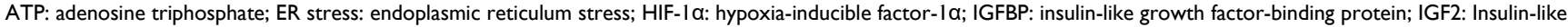
growth factor 2; mTOR: mammalian target of rapamycin; PI3K: class I phosphatidylinositol 3-kinase; ROS: reactive oxygen species; Shh: Sonic Hedgehog signaling; TFEB: transcription factor EB; ULK1: unc-51-like kinase 1. 
suppressed invasion ability $[82,83]$. Besides, Cha et al. pointed out that autophagy induced by TNF-a mediates intrinsic apoptosis via Atg5, leading to cell death [75]. Hence, autophagy regulates the functional loop between TNF- $\alpha$ and NF-kB in order to regulate trophoblastic invasion and apoptosis via MMP-2 and MMP-9. The exact role of autophagy in determining the fate and invasion ability of trophoblasts is inconsistent between these reports [80, 82, 83], which demands for further confirmations.

Mitochondrion is of great significance in cell motion and has been recognized as a regulator in migration/invasion of tumor cells and trophoblasts [84, 85]. Invasion-defective human EVTs present disordered mitochondrial function with enhanced fission, decreased respiratory and membrane potential [84]. Recent studies have worked on the role of autophagy in trophoblast invasion from the perspective of mitochondria. In primary human trophoblasts, aberrant autophagy might lead to impaired mitophagy (decreased PINK1) and disordered mitochondrion fission/fusion (decreased OPA1, MFN1, Drp1, and FIS1) [50]. Besides, autophagy regulates mitochondrial transmembrane potential, mitophagy and mitochondrial biogenesis through BNIP3 [62, 86], which is an autophagy inducer. SIRT1/AMPK/PGC-1a has been identified as an axis for governing mitochondrial biogenesis. In mouse myoblast cell line, human placental hydrolysate could rescue mitochondrial damage via autophagy through manipulating SIRT1/AMPK/ PGC-1a [87]. Whether this result applies to trophoblasts deserves more research. Anyway, impaired autophagy might lead to dysfunctional mitochondria. Interestingly, co-culture with human placenta-derived mesenchymal stem cells increases mitophagy (upregulated PINK1, ARKIN) in trophoblast cells and enhances invasion ability with increased MMP-2 and MMP-9 [88]. In all, there could be association between mitochondrial homeostasis and invasion ability of trophoblasts via mediating autophagy.

HIF-1a is another pivotal factor that mediates trophoblasts invasion via autophagy [89]. Moderately upregulated HIF-1a serves as an energy source for EVT invasion via activating autophagy. Whereas, persistent overexpression of HIF-1a may impair autophagy in EVTs, which could be rescued by supplementation of ATP [61]. Besides, the expression of HIF-1a is directly regulated in a NF-kB-dependent manner [90], rendering NF-kB-HIF-1a-MMP-2/9 as an important axis for autophagy to regulate trophoblastic invasion.

\section{Implantation}

Autophagy has also been demonstrated to participate in implantation [91], during which $\mathrm{PI} 3 \mathrm{~K} / \mathrm{Akt} / \mathrm{mTOR}$ is increased in luminal epithelium, decidual cells, embryoblast and trophoblast cells [92]. In mice, mTOR reaches the peak during the period of 'implantation window'. By contrast, administration of rapamycin, a mTOR blocker, could reduce implantation sites, highlighting the necessity of mTOR for implantation [93]. Compared with inter-implantation sites, Atg5, Atg12, LC3, cathepsin B, p62 and autophagosomes are significantly decreased at implantation sites [94]. Studies above show inhibited autophagy during implantation, however there are some challenging results. Knockout of FIP200 in mice reproductive tract leads to implantation failures. FIP200 reverses the proliferation-blockage effect mediated by progesterone and thus facilitates endometrial receptivity [13]. Particularly, autophagy might favor survival of blastocysts in delayed implantation model in mice [95].

\section{Autophagy regulates placentation}

Placenta is composed of decidua basalis, chorion frondosum and amniotic membrane. And it helps maintain pregnancy via providing oxygen, nutrients and immune defense for fetus. Based on trophoblast and other cells (fibroblasts, immune and vascular cells etc.), placental development goes through several hallmark events: implantation, trophoblastic differentiation and migration/invasion, decidualization, angiogenesis and vascular remodeling [96]. So far, it has been reported that autophagy might involve in placentation (Figure 3A and 3B). Expression levels of Atg5, Atg7 and Atg16L1 persistently increase during mice placental development, while LC3-II is scarcely detectable in placentae [97]. Actually, LC3 family members differentially express during murine placentation, among which LC3B is the most susceptible to nutrient deprivation [43]. There are several gestational complications tightly related to impaired placentation like PE, FGR, and SM, for which autophagy could be a regulator in pathogenesis. For example, LC3B-II, LAMP-2 and Beclin-1 increases in FGR placenta but mTOR and p62 declines [36, 98]. Moreover, enhanced autophagy could inhibit transplacental amino acid transport via decreasing mTOR activity [37, 99]. Folate deficiency also activates autophagy and thus jeopardizes placentation presented with smaller placenta and disordered endocrine function. Fortunately, impaired trophoblastic invasion ability could be reversed by 3-MA treatment [100]. Induced autophagy could inhibit miR-30a-5p that targets Beclin-1 and cause 
pregnancy losses. Meanwhile, morphological alterations of mice placentae include reduced villi, aberrant aggregation of trophoblast cells, atrophy of interstitial and impaired vascular structure [101]. However, there are some conflicting results display a different relationship between autophagy and placentation [44, 102]. For instance, Atg2B gene is hypermethylated and downregulated in placenta of small-for-gestational-age new-born [102]. Additionally, nutrient deprivation disturbs placental vascular remodeling system via suppressing autophagy and ER stress [44]. These studies imply that placentation may work in a compensatory way to warrant fetal growth and inhibited autophagy could be an adaptive response to insufficient nutrient supply.

From the beginning of implantation, the trophoblast stem starts to differentiate into outer layer of primitive STBs and inner layer of CTBs, during which autophagic flux is activated [97]. When implantation is completed, trophoblast develops into
VTs and EVTs, which further mediates placental development. Arikawa et al. have reported that autophagy facilitates trophoblast differentiation through downregulating Galectin-4 to establish maternal-fetal interface [103]. Defects of placentation in high glucose may attribute to ROS-induced autophagy via activating Nrf2 pathway and aberrantly increased expression of differentiationrelated gene expressions (HAND1, MASH2, PL1, MMP12 and IGF2) [104]. During differentiation, overexpression of p53 downregulates the level of LC3B-II, indicating p53 as a negative regulator of autophagy in trophoblast [39]. Interestingly, p53 also serves as a functional link in the crosstalk between autophagy and apoptosis, regulating turnover and homeostasis of trophoblast cells [105]. Placentaspecific protein 8 (PLAC8) co-localizes with p53 and assists its degradation. Autophagy induced by PLAC8 facilitates the viability and proliferation of trophoblasts and this effect can be abrogated by
A

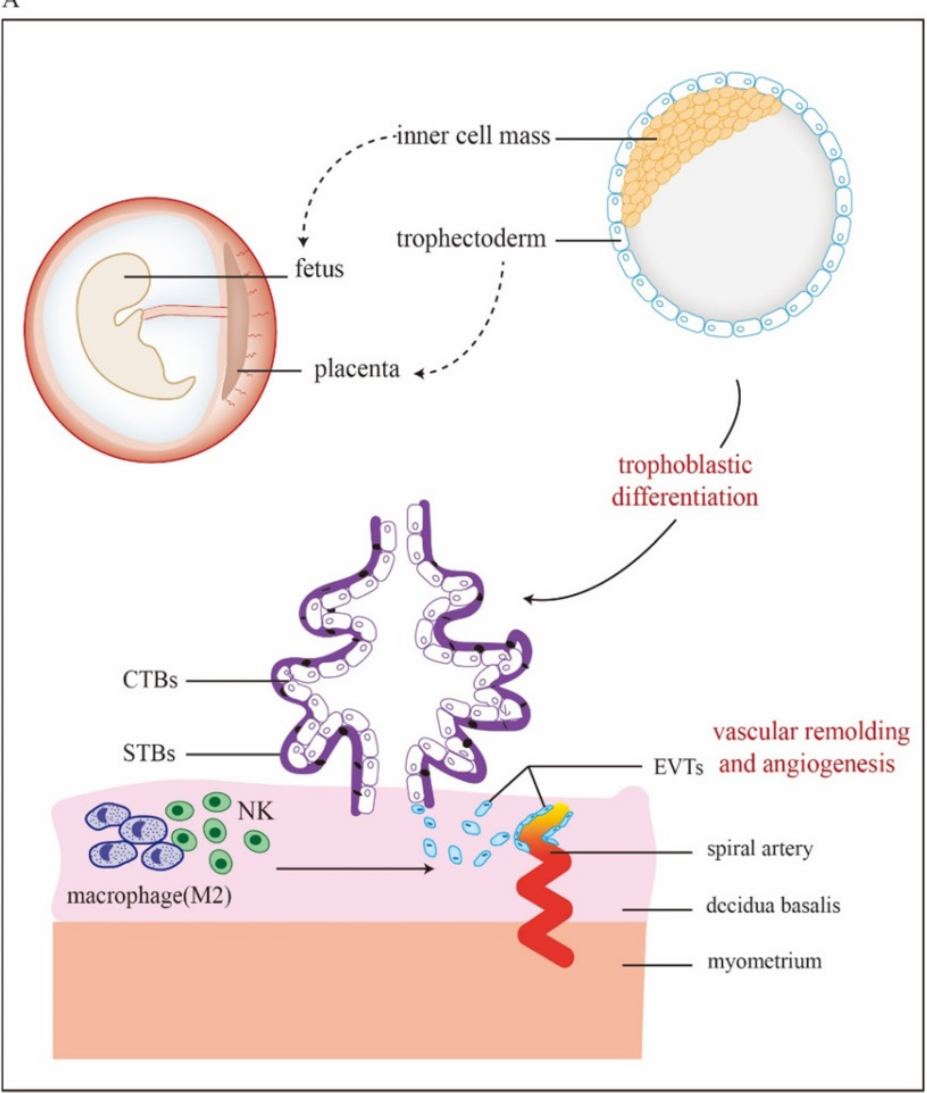

B

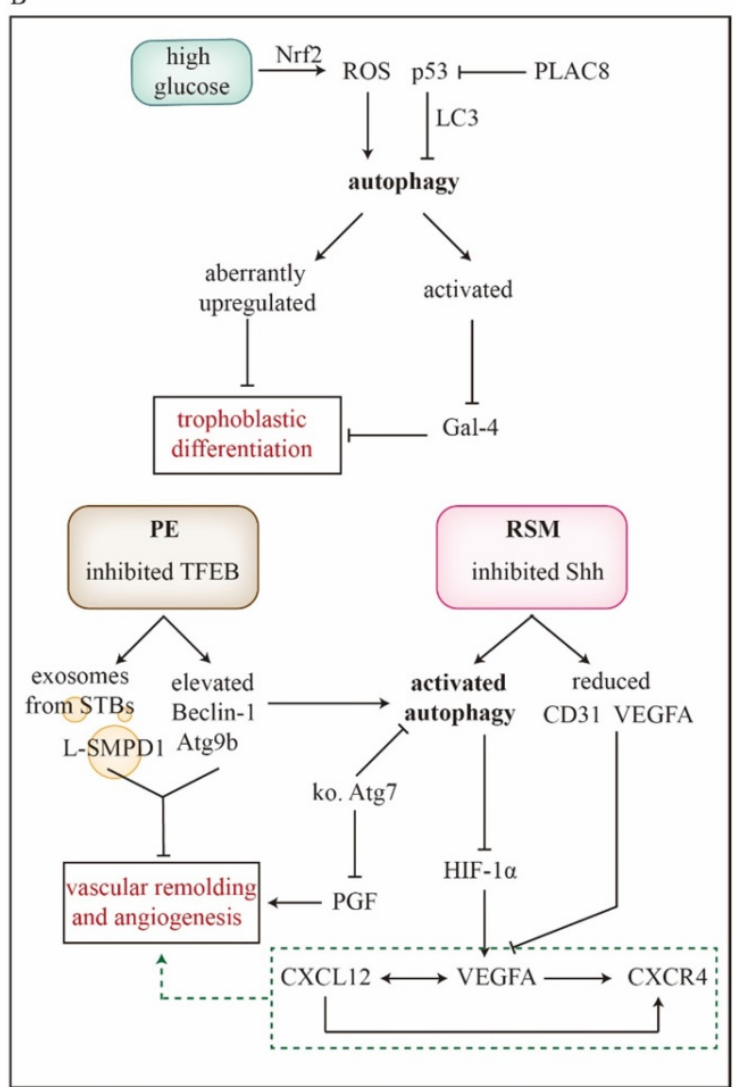

Figure 3. Roles of autophagy in trophoblastic differentiation, placental vascular remodeling and angiogenesis. A) Trophectoderm of blastocysts will further develop into placentae. In this process, trophoblasts go through differentiation into VTs and EVTs, which response for villus formation and vascular remodeling/angiogenesis, respectively. Additionally, decidual macrophages and NK cells can also facilitate placental vascular remodeling. B) The detailed mechanisms how autophagy regulates trophoblastic differentiation, placental vascular remodeling and angiogenesis are focused both in stress and pathological conditions (PE and RSM). In PE pathogenesis, TFEB is inhibited and thus upregulates Beclin-1 and Atg9b as well as promotes the exosomes containing L-SMPD1 releasing from STBs. By which way, vascular remodeling is impaired. Shh pathway, downstream of Akt, is suppressed in RSM. Moreover, it presents with activated autophagy and reduced VEGFA. Importantly, the CXCL12-VEGFA-CXCR4 axis is involved in angiogenesis, during which VEGFA relates to autophagy via the regulation by HIF-1a. Abbreviations: CTBs: cytotrophoblasts; CXCL12: C-X-C motif chemokine

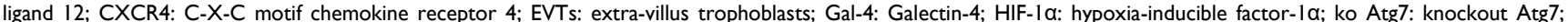
L-SMPD1: lysosomal sphingomyelin phosphodiesterase; NK cell: decidual natural killer cell; PE: preeclampsia; PGF: placental growth factor; PLAC8: Placenta-specific protein 8; ROS: reactive oxygen species; RSM: recurrent spontaneous miscarriage; Shh: Sonic Hedgehog signaling; STBs: syncytiotrophoblasts; TFEB: transcription factor EB; VEGF: vascular endothelial growth factor; VEGFA: vascular endothelial growth factor $A$. 
autophagy inhibitor chloroquine. In contrast, re-expression of p53 could partly reverse the PLAC8induced autophagy activity [106]. These findings flag the significance of autophagy in trophoblast differentiation during placental development.

Spiral artery modifications are conducted by EVTs. Interstitial trophoblasts penetrate the decidua and adjacent myometrium, and their aggregation around the arteries makes preparation for invasion of endovascular trophoblasts to build low-resistance blood flow at maternal-fetal interface. During that time, the endovascular trophoblasts form a plug at the terminal of spiral arteries until full utero-placental circulation is established at the end of first trimester [107]. The conditional knockout of mice placental Atg7 shows downregulated placental growth factor with inhibited invasion and vascular remodeling of EVTs [78], suggesting necessity of autophagy for physiological placentation. However, autophagy is also involved in other pathological conditions. In RSM, inhibited Shh pathway might enhance autophagy and reduce vascular endothelial growth factor A (VEGFA) and CD31 expression, leading to aberrant placental angiogenesis [20]. TFEB is a master transcriptional regulator of lysosomal biogenesis and autophagy. Decreased TFEB inhibits lysosomal protein expression and activates mTORC1 in autophagy-defective EVTs [41]. Of note, TFEB also elevates autophagy of PE development via exosomes. For one thing, Beclin-1 and Atg9b, both of which are downstream targets of TFEB, increase in the STBs. For another, exosomes containing active lysosomal sphingomyelin phosphodiesterase 1 (L-SMPD1) forms at the membrane of STBs and is released into maternal circulation. As a result, circulating L-SMPD1 might cause impaired placental angiogenesis [108]. Autophagy induced by inhibited protein kinase $C \beta$ represses VEGFA-mediated human umbilical vein endothelial cells tube formation [109]. Studies above imply that autophagy dysregulates placental angiogenesis. Aberrant alterations in immune cells induce impaired placentation as well. Poor vascular remodeling of placentae could be observed in NK cell depletion mice [21]. Nakabayashi et al. have clarified that populations of decidual immune cells in PE placenta, including decidual $\mathrm{CD}^{+} \mathrm{T}$ cells, $\mathrm{CD}^{+} \mathrm{T}$ cells, $\mathrm{CD}^{+} \mathrm{T}$ cells, Foxp3 ${ }^{+}$Treg cells, CD $56^{+} \mathrm{NK}$ cells, and $\mathrm{CD} 68^{+}$macrophages, are smaller than those of control. Meanwhile, p62 is significantly higher in EVTs of PE [110]. Additionally, changes in mitochondria are associated with vascular remodeling during first trimester. Mitochondrial respiration decreases at 11 weeks and the content increases at 12-13 weeks, which parallels to onset of utero-placental circulation. Increased respiration might be an adaptive response to ischemia-reperfusion [111]. In PE placentae, three major mitochondrial malfunctions are observed, including distorted mitochondrial fusion, disordered mitophagy and diminished mitochondrial biogenesis $[62,112]$. Anyway, these reports indicate the significant role of autophagy in angiogenesis and vascular remodeling during placentation.

Particularly, C-X-C motif chemokine ligand 12 (CXCL12) and C-X-C motif chemokine receptor 4 (CXCR4) signaling pathway might regulate angiogenesis via autophagy to ensure placental development. CXCL12 upregulates expression of VEGFA, which can further stimulate CXCL12 synthesis and increase secretion of CXCR4, a receptor for CXCL12. Hence, it's a positive feed-back loop with great potential to favor angiogenesis [113]. CXCL12 inhibition leads to a decrease of VEGFA and HIF-1a. Meanwhile, autophagy in endometrium is increased via suppressing Akt/mTOR [114]. Besides, HIF-1a could bind with VEGFA promoter to stimulate its expression [115]. These results may unveil how CXCL12-CXCR4 axis regulate angiogenesis via modulating autophagy at maternal-fetal interface. However, CXCL12 inhibition increases CD34 ${ }^{+}$ hematopoietic stem cells [114]. Whether the greater recruitment is a compensation for aberrant angiogenesis needs more research.

\section{Autophagy and decidualization}

\section{Level of autophagy during decidualization}

Decidua is composed of decidual stromal cells (DSCs), glands, immune cells, blood and lymph vessels, among which DSCs take up a major part. Decidualization refers to a process that endometrium highly develops into secretory lining via continuous stimulation of estradiol (E2) and progesterone (P4), making preparations for the implantation of blastocyst [116]. Subsequently, immune cells infiltrate and endometrial stromal cells (ESCs) differentiate into DSCs with accumulation of glycogen and lipid droplets. In this process, a series of proteins, cytokines and growth factors are secreted. For instance, insulin-like growth factor-binding protein 1 and prolactin, both of which are widely-used markers for well-established decidualization [117], positively associated with cyclic adenosine monophosphate (cAMP) which is an inducer of decidualization.

Regarding decidualization is energy-dependent for its frequent metabolism and biosynthesis, autophagy may server as an energy resource for metabolites recycling. According to Oestreich et al., autophagy is negatively correlated with level of ATP during human decidualization. The positive regulators of autophagy, acetyl-CoA carboxylase, 
phosphorylated-ACC and p-ULK1 are increased in decidua [118]. There also shows increased LC3-II and decreased p62 in decidua when compared with proliferative or secretory phases of endometrium tissues [94, 119]. In contrast, administration of 3-MA, impedes decidualization of mice [94]. Likewise, our previous research has also revealed upregulated LC3, LC3II/I and decreased p62 in DSCs. However, in SM patients, autophagy of DSCs is suppressed with poor dNK residence, which could be alleviated by rapamycin [21]. After treatment of bafilomycin A1, LC3B-II tends to increase, suggesting enhanced autophagy flux [118]. Similar result is also confirmed by chloroquine [120]. Studies above indicate dynamic autophagy flux is activated during human ESC decidualization. Table 1 shows the expression of autophagy-related markers on decidua or DSCs.

\section{Regulation of autophagy in decidual stromal cell}

Transcription factors homeobox A10 (HOXA10) and forkhead box $\mathrm{O} 1$ (FOXO1) are positive regulators for decidualization [121]. PI3K/Akt is activated during poor decidualization of ESCs derived from endometriosis, leading to decreased insulin-like growth factor-binding protein 1 via downregulating nuclear FOXO1 [122]. FOXO3, another member of FOXO family, shares the ability of inhibited by Akt [123]. Activation of FOXO3 induces autophagy flux and its inhibition exerts opposite effect, which has been verified in kidney [124] and neural stem cells [125]. Similarly, Akt/MMP and FOXO3a/autophagy flux are downregulated in defective DSCs when exposing to microgravity [126]. HOXA10, a decidual marker, has been reported to interact with autophagy via cathepsin $\mathrm{L}$ which degrades autolysosomes. This interaction could be inhibited by folate-deficiency via dysregulating AMPK/mTOR pathway, thereby leading to impaired decidualization of mice [127]. On top of inhibiting autophagy, folate deficiency represses apoptosis of DSCs through downregulating the ratio of Bax to Bcl-2 induced by ROS in early pregnancy [128]. Anyway, folate deficiency impairs autophagy of DSCs via manipulating AMPK/mTOR, leading to defective decidualization.

Decidualization is primarily mediated by E2 and P4 through interacting with progesterone receptor [129]. Oppositely, high insulin pregnant mice present defective decidualization and show reduction in serum E2, P4, FSH and LH levels [130]. Interestingly, Shakeel et al. have proposed that progesteroneassociated membrane component 1 could bind with LC3 and is necessary for degradative activity of autophagy in lung cancer and kidney cell lines [131]. Actually, in endometrium, both autophagy and apoptosis increase significantly during secretory phase, peaking at late-secretory phase [132]. We have also found that P4 induces autophagy of ESCs at secretory phase while E2 takes an inhibitory effect [133]. These results imply P4 might be a key factor for upregulated autophagy during decidualization. However, it seems that P4 doesn't involve in impaired decidualization induced by high-fat/high-sugar diet. High-fat/high-sugar diet induces impaired autophagy and markers for well-established decidualization (insulin-like growth factor-binding protein 1 and prolactin) are lower expressed in primary human ESCs without alterations in serum level of P4 [118]. Additionally, treating DSCs with mycophenolic, an immunosuppressive drug, autophagic flux and senescence were dramatically activated in response to nuclear stress, presented with a reduction in pre-rRNA synthesis and disruption of the nucleolus. Subsequently, p53 is stabilized by nuclear stress and thus generates a p21-mediated cell cycle arrest in late $S$ and G2 phases, inhibiting proliferation of DSCs [134]. Considering we have discussed p53 as a negative regulator of autophagy before, it could be surmised that autophagy of DSCs during decidualization might be regulated in a nuclear stress/p53/autophagy flux loop to reduce the cellular damage caused by stress.

\section{Autophagy facilitates decidualization}

As mention above, it's reasonable to deduce that autophagy probably facilitates decidualization during early pregnancy. In contrast, autophagy inhibition induced by 3-MA results in abnormal decidualization with smaller robust deciduomas, decreased uterine horn gross weight and unclear cellular boundaries [94]. Remarkably, autophagy-related proteins work differentially in mechanism. As for FIP200 which makes up ULKI complex, it mediates P4-induced decidualization. Depletion of FIP200 impairs decidualization and endometrial receptivity both in mice and human ESCs [13]. After initiation of autophagy, Atg12 is activated by Atg7 then is transferred to interact with Atg10, and forms Atg12-Atg5 complex finally. With conjunction of Atg16 or Atg16L1, an exquisite complex is established for elongation of phagophores [17, 135]. Nevertheless, Atg5, Atg7 and Atg16L1 are not entirely similar in their role of regulating autophagy during decidualization. Interestingly, knocking out Atg5 has a stronger effect on blocking autophagy flux in ESCs than knocking out Atg7. But silencing Atg7 is more effective in impairing decidualization [120], suggesting Atg7 might facilitate decidualization in an autophagy-dependent manner. Moreover, expression level of Atg16L1 remains consistent during 
decidualization, while knocking out ATG16L inhibits LC3B-II level [119]. Anyway, these results may indicate positive relationship between autophagy and decidualization. However, high insulin disturbs endometrial angiogenesis and decidualization via enhancing autophagy (increased Atg5, Beclin-1 and decreased p62) [130]. Whether elevated autophagy could be a compensation for impaired decidualization in response to insulin and the underlying mechanism still needs more supports.

\section{Autophagy and decidual immune cells}

\section{Decidual NK cell}

Natural killer (NK) cell, a cytotoxic lymphocyte, plays important roles in both innate and adaptive immune response, and induces apoptosis via releasing granzyme $\mathrm{B}$ and perforin or mediating Fas/FasL pattern. The phenotypes and activity of NK are modulated during its development and maturation, and CD3-CD56 $6^{+}$has been identified as the specific marker for NK in adults [136]. Of note, expression levels of CD56 are closely related to NK cytotoxicity. CD56 dim NK takes a percentage over 90 in total NK population and mainly exists in peripheral blood with highly expressed CD16, which facilitates antibody-dependent cellular cytotoxicity. While CD56 bright $\mathrm{CD}^{-} 6^{-} \mathrm{NK}$ is dominant in secondary lymphoid tissues and endometrium, which secrets abundance of cytokines, for instance TNF- $\alpha$, INF- $\gamma$, IL-12, IL-15 and IL-18, in order to regulate immune microenvironment [136, 137]. Additionally, the activity of NK is determined by the functional balance between its inhibitory (KIR, NKG2, etc.) and stimulating (FCGR3, NCR, etc.) receptors [138]. Dysregulation of phenotypes and cytotoxicity of NK could contribute to a series of diseases, including malignancy, infectious diseases, and endometriosis [139-141].

Decidual NK (dNK) cells are recruited in decidua, migrating through DSCs with the assistance of various chemokines like CXCL10 and CXCL12. Meanwhile, the expression profiles of chemokine receptors on $\mathrm{dNK}$ are altered in coculture with DSCs [142]. Besides, dNK cells could differentiate from uterine hematopoietic progenitor cells as well as directly derive from $\mathrm{CD} 16^{+}$peripheral blood NK cells [143]. Though there is a declining trend throughout gestation, dNK is dominant among decidual immune cells during early pregnancy [144]. Autophagy could regulate the crosstalk between $\mathrm{dNK}$ and DSCs at maternal-fetal interface [145]. Our recent research shows increased autophagy of DSCs promotes NK residence mediated by MITF-TNFRSF14/HVEMMMP9-adhension molecules axis during decidualization. However, SM patients display insufficient
dNK cell residence and impaired autophagy of DSCs. Despite dNK presents with higher level of autophagy than that of NK from peripheral blood, it doesn't regulate its own adhesion and residence directly. We also found NK depletion mice presented poor vascular remodeling [21]. Actually, dNK is also capable to induce decidual angiogenesis owing to secretion of various isoforms of vascular endothelial growth factor [146], but whether it's autophagydependent remains unclear. In all, autophagy of DSCs could facilitate dNK residence in order to build fully functional decidua.

dNK enhances trophoblast invasion and promote vascular remodeling in early pregnancy [146, 147]. Admittedly, autophagy mediates subtle connection between $\mathrm{dNK}$ and trophoblast cells. Autophagy-deficient trophoblasts increases the cytotoxicity of NK cells via upregulating IGF-2 and impedes trophoblasts invasion by downregulating paternally expressed gene 10 [148]. Population of dNK is smaller in PE placenta which might account for aberrant vascular remodeling of EVTs [110]. Liu et al. have demonstrated that UL16-binding protein 1 interacts with NKG2D receptors on dNK, which downregulates the expression level of NKG2D and thus alters the balance of cytokine secretion with elevated TNF- $\alpha$, IFN- $\gamma$, TGF- $\beta 1$, IL6 and IL-8. Subsequently, this imbalance contributes to impaired invasion of EVTs [149]. Recently, they have further reported that UL16-binding protein 1 might impair trophoblast invasion depending on TNF-a secreted by dNK, which activates autophagy via suppressing NF-KB [83].

Studies of autophagy and uterine NK (uNK) based on ESCs may offer us more ideas in exploring DSCs. The axis of autophagy/STAT3/HCK in ESCs has been reported to regulate the cytotoxicity of $\mathrm{uNK}$. In detail, autophagy suppression reduces HCK via downregulating STAT3 and thus upregulates its downstream CXCL8/IL-8 and IL-23A, resulting in increased FCGR3- NK cells. PTGS2, which is targeted by miR1185-1-3p, also upregulates FCGR3- NK cells in endometriosis [150]. Ectopic lesions of endometriosis secret abundant IL-15, which inhibits apoptosis but promotes invasion, viability and proliferation of ESCs in an autocrine manner. Meanwhile, excessive IL-15 blocks cytotoxicity of NK cells with decreased granzyme B, IFN- $\gamma$, activating receptor natural-killer group 2D (NKG2D) and natural cytotoxicity receptor NKp44. Rapamycin, an autophagy inducer, reduces the expression of IL-15 receptors on ESCs, such as IL-15Ra and IL-2R $\beta$, indicating its therapeutic potential in treating endometriosis [151]. Protopanaxadiol could reverse the inhibitory effect mediated by E2 on autophagy of ectopic ESCs and 
induce NK cytotoxicity. The expression of NKG2A, activated natural cytotoxicity receptors and IFN- $\gamma$ are dramatically elevated on NK with decreased IL-10 level [152]. Whether these regulatory mechanisms still fit in dNK and DSCs needs further confirmation.

\section{Decidual macrophage}

Macrophage, mostly derived from peripheral monocytes, residents in various organs and tissues, including decidua. During early pregnancy, macrophage takes up the second largest population of decidua immune cells, only secondary to dNK [144]. Autophagy is of great significance for the differentiation and survival of monocytes. Macrophage colony-stimulating factor (M-CSF) facilitates macrophages differentiating from monocytes and remarkably activates AMPK via ROS produced in a M-CSF receptor-dependent way. Then, enhanced AMPK induces autophagy flux in a FOXO3-dependent manner [153]. Autophagy could also be induced via freeing Beclin-1 from Bcl-2 by activating JNK and blocking Atg5 cleavage, contributing to monocyte-macrophage differentiation [154]. Moreover, M-CSF secreted by DSC induces CD14 $4^{\text {bright }} \mathrm{CD} 163^{+} \mathrm{CD} 209^{+} \mathrm{CD} 86^{\text {dim }}$ phenotype of decidual macrophage and promotes macrophage survival [155].

According to functional characteristics, macrophages could be divided into two main groups: M1 and M2. M1 macrophage, classically activated through lipopolysaccharides and IFN- $\gamma$, generates abundance of IL-12 to mediate inflammation. M2 macrophage, alternatively activated, secrets anti-inflammatory cytokines IL-10 [156]. IL-10 mediates the production of VEGF for tissue repairing and vascular remodeling under hypoxia [157]. Co-culture with DSCs makes M1 shift to M2 phenotype[158], and other studies have also verified decidual macrophage as M2 phenotype owning to its role in tissue repairing and remodeling $[144,159]$. The recruitment of M1 or M2 could be modulated by various chemokines and signaling pathways. For example, M1 is summoned by CCL19/CCL21 which could be blocked by PI3K [160], indicating the potential of autophagy in facilitating M2 polarization and residence in decidua. Of note, aberrant polarization of M1-M2 may account for several pregnancy complications, such as RSM, FGR and PE, for its role in implantation, decidualization and angiogenesis [161]. HIF-1a directly binds to VEGF transcription promoter and upregulates VEGF which induces recruitment and polarization of decidual M2 under hypoxia $[115,158]$. Zhang et al. have elucidated that inhibited hypoxia-induced autophagy downregulates HIF-1a/STAT3 with decreased VEGF [162].
In addition, autophagy might promote placental angiogenesis through VEGF via modulating CXCL12/CXCR4 as discussed before. These findings indicate that autophagy might positively regulate polarized M2 and be responsible for placental angiogenesis.

Decidual macrophage, mediating immunosuppressive effect at maternal-fetal interface, secrets IL-10 and expresses indoleamine 2,3-dioxygenase (IDO) to impede activation of $\mathrm{T}$ cells. Moreover, aberrant subgroups of decidual $\mathrm{T}$ present elevated regulatory $\mathrm{T}$ (Treg) cells and decreased naïve $\mathrm{T}$ cells [163]. IL-10, an anti-inflammatory cytokine, is verified to reduce among RSM patients [164], with alteration in both its promoter and intron polymorphisms [165]. As for murine macrophage, IL-10 inhibits the activation of autophagy via PI3K signaling pathway [166]. In asthma, inhibiting autophagy of airway macrophages is entitled to induce immunosuppressive effect mediated by IL-10 [167]. These results indicate that IL-10 produced by decidual macrophage might decrease and thus impair immune tolerance in RSM patients. IDO, which is also expressed by EVTs, maintains immune tolerance by suppressing T-cell response [168]. IDO expression could reduce embryo absorption rate via increasing Treg and alleviating inflammatory response in RSM models [169]. However, there are not relevant articles directly about autophagy of decidual M2 macrophage in regulating maternal immune tolerance, and the relationship among IDO, autophagy and immune regulation seems complicated in other cell lines and samples. IDO-mediated immunosuppression activates mTOR signaling pathway to facilitate T-cell tolerance [170]. Upregulated IDO induced by IFN- $\gamma$ could dramatically enhance autophagy and activate phagocytosis of macrophage, inhibiting growth of cervical cancer cells [171]. Whereas, Chinnadurai et al. have reported that IDO-induced immunosuppression works in an autophagy-independent manner [172]. Taken together, decidual macrophages are involved in maintaining immune tolerance via upregulating Treg, whether autophagy influences this process and how IDO and IL-10 regulate immune milieu at maternal-fetal interface still remain elusive.

\section{Decidual $\mathrm{T}$ cell}

Decidual $\mathrm{T}$ cells regulate maternal-fetal immunity in an intricate way for their various functions and phenotypes. In early pregnancy, 30-45\% of decidual $\mathrm{T}$ cells are $\mathrm{CD} 4^{+} \mathrm{T}$ cells and $45-75 \%$ are $\mathrm{CD}^{+} \mathrm{T}$ cells. $\mathrm{CD}^{+} \mathrm{T}$ cells could be decided into Th1, Th2, Treg and Th17 subgroups based on their transcription expression factors and secreted cytokines. They comprise $5-30 \%, 5 \%, 5 \%, 2 \%$ of total 
population of decidual $\mathrm{CD} 4^{+} \mathrm{T}$ cells respectively, and are differently regulated in order to maintain pregnancy [173]. The axis of Th1/Th2 is regarded as a classical pattern that is involved in maternal-fetal immune tolerance. Th1 is dominant during pre-implantation period and favors trophoblast invasion, after that Th2 takes over to warrant optimal conditions for fetal development and placentation. Tilted balance towards Th1 contributes to pregnancy disorders, such as PE and RSM [174]. Actually, cytokines generated by Th1 and Th2 could further influence the level of autophagy. Autophagy is enhanced by IFN- $\gamma$ and TNF- $\alpha$ fromTh1, and is suppressed by IL-4, IL-13 and the anti-inflammatory cytokine IL-10 from Th2 [175]. Treg $\left(\mathrm{CD} 4{ }^{+} \mathrm{CD} 25^{+}\right.$ Foxp $3^{+}$) cells are recruited before implantation period and produce immunosuppressive cytokines like IL-9, IL-10 and TGF- $\beta$ to help build immune tolerance. Besides, Treg cells can be induced by VEGF milieu of M2 through increased programmed death-ligand 1 (PD-L1) [176]. Of note, PI3K/mTOR, an inhibitory pathway upstream of autophagy, has been reviewed as a regulator in development, function, and stability of Treg cells [177]. Th17 is negatively associated with female fertility [178], and its related cytokines (IL-23, IL-17, IL-6) upregulate in SM [179]. Whereas, no statistically significant polymorphic site of IL-17A/F is observed for susceptibility of SM [180]. Particularly, autophagy has been reported to protect organ injuries caused by Th1 and Th17 in sepsis [181], indicating autophagy might balance Th1 and Th17. Anyway, both Th1/Th2/Th17 and Treg paradigms modulate immunological milieu in normal pregnancy and several pregnancy complications [182]. Though the role of autophagy in decidual $\mathrm{T}$ cells during gestation has not been reported so far, we could still get some insights from context.

Maternal E2 and P4 exert pivotal modulatory properties on initiation and maintenance of conception, which also elicits polarization of $\mathrm{CD}^{+} \mathrm{T}$ cells. P4, which is involved in implantation and decidualization, upregulates Th2, Treg and downregulates Th1 and Th17. E2, which is involved in endometrium receptivity, promotes Treg and Th2 while suppresses Th1, probably through TGF- $\beta$-mediated IDO expression [178, 183]. Besides, it has been well described that E2 and P4 regulate autophagy through interacting with estrogen receptors (ER) and progesterone receptors (PGR), respectively [10, 184, 185]. In fact, ER and PGR have been verified to express on $\mathrm{T}$ lymphocytes in human, and downregulation of their receptors is tightly correlated with SM [186]. However, there are few reports about effects of E2 and P4 on autophagy of T lymphocytes yet. Taken together, ovarian hormones might regulate polarization of $\mathrm{CD}^{+} \mathrm{T}$ cells mediated by autophagy via ER and PGR, which still remains to be confirmed by further studies.

Transforming growth factor beta (TGF- $\beta$ ) regulates proliferation and differentiation of $\mathrm{T}$ cells [187] and corporates with IL-10 to suppress Th cells [182]. TGF- $\beta /$ SMAD signaling is associated with pregnancy and fertility for its role in endometrium receptivity, implantation [188], proliferation and invasion of trophoblasts [189] and decidualization [190]. Notably, emerging research suggest TGF- $\beta$ could induce autophagy with increased Beclin-1, Atg5 and Atg7 in other cell lines [191, 192]. Thereby, autophagy is supposed to modulate decidual $\mathrm{T}$ cells by means of TGF- $\beta$ during pregnancy. Chronic endometritis patients usually suffer from recurrent implantation failures and their serum present higher levels of IL-17 but lower levels of IL-10 and TGF- $\beta$ in endometrium. Simultaneously, autophagy locates in ESCs where LC3 increases with inhibited mTORC1 [193]. That indicates autophagy in ESCs may disturb the endometrial receptivity, immune milieu and implantation via mediating shift from Treg towards Th17. Soluble endoglin produced by trophoblasts not only inhibits autophagy but also dysregulates the differentiation of Treg cells by downregulating TGF- $\beta$, participating in the development of PE with invasion failure of trophoblasts [194]. Moreover, TFEB, an inducer of autophagy, is critical for TGF- $\beta$-induced migration and metastasis of pancreatic cancer cells [195]. TFBE also promotes releasing of ICAM-1, a molecule for T cell adhesion, to impair placental angiogenesis via exosomes [108]. Whether autophagy regulates decidual $\mathrm{T}$ cell adhesion via TGF- $\beta$ and how it modulates crosstalk among decidual T cells, trophoblasts and ESCs/DSCs should be an interesting topic to explore.

\section{Autophagy disorder and spontaneous miscarriage}

\section{Aberrant autophagy in SM patients}

Around $15 \%$ pregnancies end up with SM, which is characterized by pregnancy loss before the $20^{\text {th }}$ week. In particular, RSM refers to occurrence of 3 or more consecutive miscarriages, and over half of cases show no identifiable causes [196]. Accumulating evidence indicates the important role of autophagy because of aberrant expression of autophagy-related markers in SM/RSM (Table 2). LC3, LC3II/I, Atg5 and Beclin-1 are higher in RSM placentae [81, 197] along with inhibited Shh signaling pathways [20]. Avagliano et al. have also shown that STBs in SM exhibit a higher density of LC3 staining with more autophagosomes than those of normal controls. 
HIF-1a increases in SM villi and decidua, while the ratio of Bax to Bcl-2 and cleaved caspase 3 are elevated in decidua of $\mathrm{SM}$, without statistical significance, though. Thus, autophagy might be a protector from hypoxia-induced apoptosis [35]. Interestingly, as compared to women with their first conceptions, autophagy of peripheral blood mononuclear cells increases in women with prior spontaneous pregnancy losses but no deliveries [198]. However, here exists some discrepancies. Yang et al. have shown that plasmacytoma variant translocation 1 (PVT1) is decreased in villi from RSM patients. In vitro, PVT1 knockdown elicits impaired autophagy with increased mTOR, p62 and decreased Beclin-1, LC3II/I, ULK1 in HTR-8/SVneo cell line [199]. Our previous studies display that autophagy is impaired in RSM. Smaller number of autophagosomes with aberrant distribution are exhibited in the villi of RSM patients [148]. Insufficient autophagy with lower Atg5, LC3B and higher p62 is observed in DSCs derived from SM patients. Besides, downstream molecules of autophagy mediating NK residence are also reduced [21]. Wei et al. have confirmed these results in rat model induced by antiphospholipid antibodies. Elevated p62 and mTOR as well as downregulated Beclin-1 and LC3-II are presented in placentae and the impaired autophagy could be rescued by rapamycin [200]. Except for different ways to evaluate of autophagy, cell types and sampling, various pathogenic mechanisms involved in could still be to blame for the inconsistence. Of note, autophagy is a dynamic process and might act as a compensation for cellular stress in SM. Furthermore, the exact causal relationship between altered autophagy and SM remains to be established based on larger scale of subjects and well-designed studies.

\section{Potential therapeutic methods targeting autophagy for SM}

In normal pregnancy, Yin Yang 1 (YY1) can interact with plasmacytoma variant translocation 1
(PVT1) promoter to upregulate its transcription, which further favors trophoblasts invasion and adhesion by autophagy via mTOR signaling. Effects followed with decreased PVT1 in villi samples might be involved in RSM pathogenesis, rendering PVT1 as a therapeutic target [199]. PLAC8, a newly-explored factor in tumorigenesis, has been regarded as a target of treating oral squamous cell carcinoma for its regulatory roles in ERK[201], Wnt/ $\beta$-catenin and PI3K/Akt/GSK3 $\beta$ pathways [202], respectively. In trophoblasts, PLAC8 co-localizes with p53 and mediates its degradation, facilitating the viability, proliferation and differentiation via enhanced autophagy [106]. Punicalagin enhances autophagic flux to protect primary human STBs from apoptosis induced by stress conditions [203]. Hyperoside activates autophagy and combats inflammatory response in rat model with decreased IL-1 $\beta$, IL-8, ICAM-1, VCAM-1 and complement C3, thereby rescues pregnancy losses via downregulating mTOR/S6K and TLR4/MyD88/NF-kB [200]. Studies above offer us insights into treatment of RM targeting autophagy. Rapamycin, a mTOR inhibitor to induce autophagy, could also reverse adverse pregnancy outcomes and elevates LC3 stanning in junctional and labyrinth zones in placentae of rats [200]. It effectively induces autophagy to reduce NK cytotoxicity in trophoblasts [148] and promote DSC autophagy and NK residence in decidua [21]. Antiphospholipid antibodies-induced NLRP3-mediated IL-1 $\beta$ secretion in human trophoblasts could also be inhibited by rapamycin [204]. In folate-deficient models, rapamycin alleviates impaired autophagy and defective decidualization [127, 128]. However, rapamycin has also been reported for its potential in treating endometriosis [151] and endometriumcarcinoma [205] via enhancing NK cytotoxicity, reminding us that dose and side effects of rapamycin should be considered carefully when it comes to SM (Table 3).

Table 2. Dysregulated autophagy and alterations of related molecules in RSM/SM

\begin{tabular}{|c|c|c|c|c|}
\hline Study & Objects & Aberrant autophagy & Samples & Alterations of relevant molecules \\
\hline \multirow[t]{2}{*}{ Zhou, 2021} & human & $\uparrow$ & villi in SM & increased Beclin1, LC3II/I, HMGB1 \\
\hline & & & LPS-treated HTR-8/SVneo & \\
\hline Cai, 2018 & human & $\uparrow$ & villi in SM & decreased MFN2; increased Beclin 1, LC3II/I, Atg5 \\
\hline \multirow[t]{2}{*}{ Pan, 2021} & human & $\uparrow$ & villi in RSM & decreased Shh, PTCH, SMO, Gli1/2/3 \\
\hline & & & JAR with Shh inhibition & increased LC3B, LC3II/I, LAMP1 \\
\hline \multirow[t]{2}{*}{ Avagliano, 2015} & human & $\uparrow$ & STBs/villi in SM & increased LC3, HIF-1a \\
\hline & & & decidua & increased HIF-1a, Bax/Bcl-2, cleaved caspase 3 \\
\hline Jayaram, 2018 & human & $\uparrow$ & $\mathrm{PBMC}$ in SM & decreased p62, hsp70 \\
\hline \multirow[t]{2}{*}{ Yang, 2020} & human & $\downarrow$ & villi in RSM & decreased PVT1 \\
\hline & & & HTR-8/SVneo with PVT downregulation & increased mTOR, p62; decreased Beclin-1, LC3- II/I, ULK1 \\
\hline Tan, 2020 & human & $\downarrow$ & villi in RSM & decreased autophagosomes \\
\hline Lu, 2020 & human & $\downarrow$ & DSCs in SM & increased p62; decreased Atg5, LC3B, MITF, TNFRSF14, MMP9 \\
\hline Wei, 2020 & rats & $\downarrow$ & placentae in a-PL rats & increased p62, mTOR; decreased Beclin-1, LC3-II \\
\hline
\end{tabular}

Abbreviations: aPL: antiphospholipid; Atg: autophagy-related genes; DSCs: decidual stromal cells; HIF-1a: hypoxia-inducible factor-1a; LC3: microtubule-associated protein 1 light chain 3; MMPs: matrix metalloproteinases; mTOR: mammalian target of rapamycin; PBMC: peripheral blood mononuclear cells; PVT1: plasmacytoma variant translocation 1; RSM: recurrent spontaneous miscarriage; Shh: Sonic Hedgehog signaling; SM: spontaneous miscarriage; STBs: syncytiotrophoblasts 
To date, vitamin $\mathrm{D}$ has drawn great interests in reproductive system for its deficiency correlated with endocrine diseases and adverse pregnancy outcomes [206]. Especially for pregnancy complications presented with poor placentation, decreased vitamin D could be a risk factor [207]. A prospective cohort study conducted by Mumford et al. reports that sufficient preconception, instead of early pregnancy, concentrations of serum vitamin $\mathrm{D}$ are associated with better pregnancy outcomes among women who have suffered from one or two prior pregnancy losses before [208]. Recent research indicates autophagy may participate in interactions between vitamin $\mathrm{D}$ and placental development, but whether it's mediated by vitamin D receptor (VDR) still remains controversial. Reduction of maternal serum vitamin $\mathrm{D}$ and intracellular VDR may lead to impaired vitality (decreased LC3B/Beclin-1) and reduced ratio of VTs in placenta [209]. Additionally, the VDR protein is upregulated after supplementation without significant increase in its RNA and target gene level, indicating the importance of VDR but this effect is mediated by nongenomic response [210]. However, according to Wilson et al., though genetic deficiency of
VDR caused aberrant autophagy with greater expression of Atg4b and activated mTOR, no statistically significant differences are observed in fetal growth, overall morphological parameters in VDR null placentae in comparison to controls [211]. Vitamin D can rescue the impaired viability and invasion of trophoblasts induced by ROS-treated HTR-8 cell line [212]. Fetal mortality, organ dysfunction and metabolic disorders could be alleviated by vitamin $\mathrm{D}$ supplementation in ischemia placenta via activating autophagy and attenuating apoptosis [210]. During implantation and decidualization, vitamin $\mathrm{D}$ suppresses inflammation and positively regulates HOXA10 at maternal-fetal interface [213]. Importantly, vitamin D decreases Th17 cells as well as the ratio of Th17 to Treg in peripheral blood of SM patients [214]. Whether these effects are mediated by autophagy needs further confirmations. To be more specific, crosstalk among placenta, decidua and maternal serum vitamin D levels could play a critical role in pathogenesis of SM. Anyway, vitamin $\mathrm{D}$ should be a potential therapeutic method for $\mathrm{SM}$ in regulating immunological milieu and placentation (Table 3).

Table 3. Potential treating methods for RSM/SM targeting autophagy

\begin{tabular}{|c|c|c|c|c|}
\hline Targets & $\begin{array}{l}\text { Supporting } \\
\text { Study }\end{array}$ & Objects & Tissue/Cell line & Potential effects \\
\hline PVT1 & Yang, 2020 & human & $\begin{array}{l}\text { villi } \\
\text { HTR-8/SVneo }\end{array}$ & $\begin{array}{ll}\text { - } & \text { trigger autophagy via inactivating mTOR } \\
\text { - } & \text { facilitate invasion and adhesion ability of trophoblast cells }\end{array}$ \\
\hline PLAC8 & Feng, 2021 & human & $\begin{array}{l}\text { first trimester placentae } \\
\text { decidua } \\
\text { HTR-8 and JAR }\end{array}$ & $\begin{array}{l}\text { - } \quad \text { co-localize with p53 (an inhibitor of differentiation) and response for its degradation } \\
\text { - } \quad \text { upregulate AMPK, LC3II/I, Atg5, Atg12, and Beclin-1; downregulate p62 } \\
\text { - } \quad \text { regulate trophoblast cell cycle and proliferation via p53 and its downstream molecule p21 }\end{array}$ \\
\hline Punicalagin & Wang, 2016 & human & $\begin{array}{l}\text { primary human } \\
\text { trophoblasts }\end{array}$ & $\begin{array}{l}\text { - } \quad \text { activate autophagic flux in STBs } \\
\text { - favor STBs survival via increased autophagy }\end{array}$ \\
\hline Hyperoside & Wei, 2020 & rats & $\begin{array}{l}\text { placentae } \\
\text { in vivo }\end{array}$ & $\begin{array}{l}\text { - } \quad \text { rescue pregnancy losses, exert effect of promoting autophagy and anti-inflammation } \\
\text { - } \quad \text { decrease IL-1 } \beta \text {, IL-8, TF, ICAM-1, VCAM-1, complement C3, mTOR, p62 } \\
\text { increase LC3 and Beclin-1 }\end{array}$ \\
\hline \multirow[t]{5}{*}{ Rapamycin } & Wei, 2020 & rats & $\begin{array}{l}\text { placentae } \\
\text { in vivo }\end{array}$ & $\begin{array}{l}\text { - } \quad \text { promote autophagy and suppress the inflammation } \\
\text { - } \quad \text { elevates LC3 stanning in junctional and labyrinth zone of placentae }\end{array}$ \\
\hline & Mulla, 2018 & human & EVTs & - $\quad$ suppress aPL-induced NLRP3-mediated IL-1 $\beta$ secretion \\
\hline & $\mathrm{Lu}, 2020$ & $\begin{array}{l}\text { human } \\
\text { mice }\end{array}$ & $\begin{array}{l}\text { decidua, } \mathrm{dNK} \\
\text { placentae, uterus, } \mathrm{dNK} \\
\text { in vivo }\end{array}$ & $\begin{array}{l}\text { - } \quad \text { induce autophagy to reduce NK cytotoxicity } \\
\text { promote DSCs autophagy and mediate NK residence in decidua via } \\
\text { MITF-TNFRSF14-MMP-adhesion molecules axis }\end{array}$ \\
\hline & Zhang, 2021 & mice & $\begin{array}{l}\text { induced DSCs in vitro } \\
\text { in vivo }\end{array}$ & $\begin{array}{l}\text { - rescue impaired decidualization in folate-deficiency conditions via activating autophagy by } \\
\text { modulating AMPK/mTOR }\end{array}$ \\
\hline & Chen, 2018 & mice & DSC & increase LC3II, Bax/Bcl-2 and reduce mTOR, p62 during decidualization \\
\hline \multirow[t]{4}{*}{ Vitamin D } & Hutabarat, 2018 & human & placentae & $\begin{array}{l}\text { - corelate with MAP1LC3B/Beclin-1, involve in regulating trophoblast viability and } \\
\text { placentation }\end{array}$ \\
\hline & Tian, 2016 & rats & placentae & $\begin{array}{l}\text { - } \quad \text { attenuate placenta apoptosis } \\
\text { upregulate Beclin-1 and LC3II/I placental trophoblast cells to reverse damage in PE model }\end{array}$ \\
\hline & $\mathrm{Pi}, 2021$ & human & HTR-8 & $\begin{array}{l}\text { - } \quad \text { promote trophoblastic viability and invasion } \\
\text { - } \quad \text { rescue impaired autophagy via increasing Beclin-1 and LC3-II }\end{array}$ \\
\hline & Rafiee, 2015 & human & peripheral blood & - $\quad$ downregulate Th17/Treg ratio \\
\hline Survivin & Pan, 2020 & mice & embryo & $\begin{array}{l}\text { - } \quad \text { protect embryo from ROS-induced apoptosis and autophagy } \\
\text { - } \quad \text { regulate spindle organization and chromosome alignment }\end{array}$ \\
\hline
\end{tabular}

Abbreviations: AMPK: 5 ' adenosine monophosphate-activated protein kinase; aPL: antiphospholipid; Atg: autophagy-related genes; dNK cell: decidual natural killer cell; DSCs: decidual stromal cells; IL: interleukin; LC3: microtubule-associated protein 1 light chain 3; MMPs: matrix metalloproteinases; mTOR: mammalian target of rapamycin; PE: preeclampsia; RSM: recurrent spontaneous miscarriage; SM: spontaneous miscarriage; STBs: syncytiotrophoblasts; Th: T helper cell; Treg: regulatory T cells 


\section{Summary and future perspectives}

Herein, we review the role of autophagy in regulating critical events during gestation, including fertilization and embryonic development, bio-functions of trophoblasts, placental development, decidualization, infiltration and residence of decidual NK, macrophage, and T cells. However, studies about autophagy in decidual macrophage and $\mathrm{T}$ cells are few, which needs more investigations. In addition, underlying mechanisms of RM mediated by aberrant autophagy are included, and thus we mention relevant potential therapeutic methods for SM by targeting autophagy. Particularly, rapamycin and vitamin $\mathrm{D}$ have been hotpots in recent decade for their multiple roles in regulating trophoblast survival, decidualization, or immunological milieu. More importantly, autophagy is a dynamic process and is involved in both maintaining homeostasis and causing pathological damage under stress. As a result, autophagy could act as a compensation in certain conditions which might lead to misinterpretations for results and mechanisms. Autophagy-related molecules might be new strategies for predicting SM, though the source of sampling and its power in scanning tests are still challenging problems. For example, Atg5 is not only a key factor in promoting trophoblast survival but also of great importance in regulating decidualization, monocyte-macrophage differentiation, mediating interaction between TGF- $\beta$ and decidual T cells, possibly. Further well-designed studies are required to verify the role of autophagy in SM pathogenesis and the network of related regulatory mechanisms in order to make potential treatment methods into practice.

\section{Acknowledgements}

This study was supported by the Major Research Program of National Natural Science Foundation of China (NSFC) (No. 92057119, 31970798, 81901563, 82071646, 82001636); the Shanghai Sailing Program (19YF1438500), the Program for Zhuoxue of Fudan University (JIF157602), the Support Project for Original Personalized Research of Fudan University (IDF157014/002), and the Open Project Program of Shanghai Key Laboratory of Female Reproductive Endocrine-Related Diseases (17DZ2273600).

\section{Author Contributions}

M.Q.L. and X.Y.Q. conceived and designed the review; X.Y.Q., H.H.S., M.Q.L., W.J.Z., J.M., H.L., X.F.T., R.Z., W.H.Z., T.Z., D.J.L. and J.F.Y. drafted and revised the manuscript. X.Y.Q. and M.Q.L. generated the tables and figures.

\section{Abbreviations}

AMPK: 5' adenosine monophosphate-activated protein kinase; Atg: autophagy-related genes; ATP: adenosine triphosphate; CTBs: cytotrophoblasts; CXCL12: C-X-C motif chemokine ligand 12; CXCR4: C-X-C motif chemokine receptor 4 ; dNK cell: decidual natural killer cell; DSCs: decidual stromal cells; ER stress: endoplasmic reticulum stress; ESCs: endometrial stromal cells; EVTs: extra-villus trophoblasts; E2: estradiol; FGR: fetal growth restriction; FIP200: focal adhesion kinase family interacting protein of 200 kDa; FOXO1: forkhead box O1; HF/HS: high-fat/ high-sugar; HIF-1a: hypoxia-inducible factor-1a; HOXA10: homeobox A10; IDO: indoleamine 2,3-dioxygenase; IGF2: Insulin-like growth factor 2; IL: interleukin; LC3: microtubule-associated protein 1 light chain 3; LncRNA: long non-coding RNA; MMPs: matrix metalloproteinases; miR: micro-RNA; mTOR: mammalian target of rapamycin; mTORC1: mTOR complex 1; NF-kB: nuclear factor kappa-B; NK cell: natural killer cell; PE: preeclampsia; PI3K: class I phosphatidylinositol 3-kinase; PLAC8: Placentaspecific protein 8 ; PVT1: plasmacytoma variant translocation 1; $\mathrm{P} 4$ : progesterone; ROS: reactive oxygen species; RSM: recurrent spontaneous miscarriage; Shh: Sonic Hedgehog signaling; SM: spontaneous miscarriage; STBs: syncytiotrophoblasts; TFEB: transcription factor EB; TGF- $\beta$ : Transforming growth factor beta; Th: T helper cell; TNF-a: Tumor Necrosis Factor alpha; Treg: regulatory T cells; ULK1: unc-51-like kinase 1; uNK cell: uterine NK cell; VDR: vitamin D receptor; VEGF: vascular endothelial growth factor; VEGFA: vascular endothelial growth factor A; VTs: villus trophoblasts; 3-MA: 3-methyladenine.

\section{Competing Interests}

The authors have declared that no competing interest exists.

\section{References}

1. Rossen LM, Ahrens KA, Branum AM. Trends in Risk of Pregnancy Loss Among US Women, 1990-2011. Paediatric and perinatal epidemiology. 2018; 32: 19-29.

2. Simpson JL. Causes of fetal wastage. Clinical obstetrics and gynecology. 2007; 50: 10-30.

3. Weselak M, Arbuckle TE, Walker MC, Krewski D. The influence of the environment and other exogenous agents on spontaneous abortion risk. Journal of toxicology and environmental health Part B, Critical reviews. 2008; 11: 221-41.

4. Eschenbach DA. Treating spontaneous and induced septic abortions. Obstetrics and gynecology. 2015; 125: 1042-8.

5. Mizushima N, Komatsu M. Autophagy: renovation of cells and tissues. Cell. 2011; 147: 728-41.

6. Matsuzawa-Ishimoto Y, Hwang S, Cadwell K. Autophagy and Inflammation. Annual review of immunology. 2018; 36: 73-101.

7. Limanaqi F, Biagioni F, Gambardella S, Familiari P, Frati A, Fornai F Promiscuous Roles of Autophagy and Proteasome in Neurodegenerative Proteinopathies. International journal of molecular sciences. 2020; 21: 3028 
8. White E. The role for autophagy in cancer. The Journal of clinical investigation. 2015; 125: 42-6.

9. Shibutani ST, Saitoh T, Nowag H, Münz C, Yoshimori T. Autophagy and autophagy-related proteins in the immune system. Nature immunology. 2015; 16: 1014-24.

10. Shen HH, Zhang T, Yang HL, Lai ZZ, Zhou WJ, Mei J, et al. Ovarian hormones-autophagy-immunity axis in menstruation and endometriosis. Theranostics. 2021; 11: 3512-26.

11. Devis-Jauregui L, Eritja N, Davis ML, Matias-Guiu X, Llobet-Navàs D. Autophagy in the physiological endometrium and cancer. Autophagy. 2021; 17: 1077-95.

12. Gu NH, Li GJ, Yang BX, You M, Lin Y, Sun F, et al. Hypo-Expression of Tuberin Promotes Adenomyosis via the mTOR1-Autophagy Axis. Frontiers in cell and developmental biology. 2021; 9: 710407.

13. Oestreich AK, Chadchan SB, Medvedeva A, Lydon JP, Jungheim ES, Moley KH, et al. The autophagy protein, FIP200 (RB1CC1) mediates progesterone responses governing uterine receptivity and decidualizationt. Biology of reproduction. 2020; 102: 843-51.

14. Nakashima A, Tsuda S, Kusabiraki T, Aoki A, Ushijima A, Shima T, et al. Current Understanding of Autophagy in Pregnancy. International journal of molecular sciences. 2019; 20: 2342.

15. Wada Y, Sun-Wada G-H, Kawamura N, Aoyama M. Role of autophagy in embryogenesis. Current Opinion in Genetics \& Development. 2014; 27: 60-6.

16. Kim J, Kundu M, Viollet B, Guan KL. AMPK and mTOR regulate autophagy through direct phosphorylation of Ulk1. Nature cell biology. 2011; 13: 132-41.

17. Walczak M, Martens S. Dissecting the role of the Atg12-Atg5-Atg16 complex during autophagosome formation. Autophagy. 2013; 9: 424-5.

18. Lamark T, Svenning S, Johansen T. Regulation of selective autophagy: the p62/SQSTM1 paradigm. Essays in biochemistry. 2017; 61: 609-24.

19. Mauthe M, Orhon I, Rocchi C, Zhou X, Luhr M, Hijlkema KJ, et al. Chloroquine inhibits autophagic flux by decreasing autophagosome-lysosome fusion. Autophagy. 2018; 14: 1435-55.

20. Pan Y, Yan L, Chen Q, Wei C, Dai Y, Tong X, et al. Dysfunction of Shh signaling activates autophagy to inhibit trophoblast motility in recurrent miscarriage. Experimental \& molecular medicine. 2021; 53: 52-66.

21. Lu H, Yang HL, Zhou WJ, Lai ZZ, Qiu XM, Fu Q, et al. Rapamycin prevents spontaneous abortion by triggering decidual stromal cell autophagy-mediated NK cell residence. Autophagy. 2021; 17:2511-27.

22. Wang H, Wan H, Li X, Liu W, Chen Q, Wang Y, et al. Atg7 is required for acrosome biogenesis during spermatogenesis in mice. Cell research. 2014; 24: 852-69.

23. Song W-H, Yi Y-J, Sutovsky M, Meyers S, Sutovsky P. Autophagy and ubiquitin-proteasome system contribute to sperm mitophagy after mammalian fertilization. Proceedings of the National Academy of Sciences of the United States of America. 2016; 113: E5261-E70.

24. Gao JG, Yang JK, Zhu L, Xu C, Nie LW. Acrylamide impairs the developmental potential of germinal vesicle oocytes by inducing mitochondrial dysfunction and autophagy/apoptosis in mice. Human \& experimental toxicology. 2021; 40:S370-S380.

25. Wang $\mathrm{Y}$, Xing $\mathrm{CH}$, Zhang HL, Pan ZN, Sun SC. Exposure to nivalenol declines mouse oocyte quality via inducing oxidative stress-related apoptosis and DNA damage. Biology of reproduction. 2021; 105:1474-83.

26. Tsukamoto S, Kuma A, Murakami M, Kishi C, Yamamoto A, Mizushima N. Autophagy is essential for preimplantation development of mouse embryos. Science. 2008; 321: 117-20.

27. Ambartsumyan G, Clark AT. Aneuploidy and early human embryo development. Human Molecular Genetics. 2008; 17: R10-R5.

28. Zahedi-Amiri A, Sequiera GL, Dhingra S, Coombs KM. Influenza a virus-triggered autophagy decreases the pluripotency of human-induced pluripotent stem cells. Cell death \& disease. 2019; 10: 337.

29. Delorme-Axford E, Bayer A, Sadovsky Y, Coyne CB. Autophagy as a mechanism of antiviral defense at the maternal-fetal interface. Autophagy. 2013; 9: 2173-4.

30. Pan MH, Zhu CC, Ju JQ, Xu Y, Luo SM, Sun SC, et al. Single-cell transcriptome analysis reveals that maternal obesity affects DNA repair, histone methylation, and autophagy level in mouse embryos. Journal of cellular physiology. 2021; 236: 4944-53.

31. Pan MH, Ju JQ, Li XH, Xu Y, Wang JD, Ren YP, et al. Inhibition of survivin induces spindle disorganization, chromosome misalignment, and DNA damage during mouse embryo development. Cell cycle. 2020; 19: 2148-57.

32. Goodale LF, Hayrabedyan S, Todorova K, Roussev R, Ramu S, Stamatkin $\mathrm{C}$, et al. PreImplantation factor (PIF) protects cultured embryos against oxidative stress: relevance for recurrent pregnancy loss (RPL) therapy. Oncotarget. 2017; 8: 32419-32432.
33. Poillet-Perez L, Despouy G, Delage-Mourroux R, Boyer-Guittaut M. Interplay between ROS and autophagy in cancer cells, from tumor initiation to cancer therapy. Redox biology. 2015; 4: 184-92.

34. Pan YJ, He L, Zhou SJ, Zhang LJ, Zhang AH, Zhao YY. Expression of urotensin II is associated with placental autophagy in patients with severe preeclampsia. Journal of human hypertension. 2018; 32: 759-69.

35. Avagliano L, Terraneo L, Virgili E, Martinelli C, Doi P, Samaja M, et al. Autophagy in Normal and Abnormal Early Human Pregnancies. Reproductive sciences. 2015; 22: 838-44.

36. Curtis S, Jones CJ, Garrod A, Hulme CH, Heazell AE. Identification of autophagic vacuoles and regulators of autophagy in villous trophoblast from normal term pregnancies and in fetal growth restriction. The journal of maternal-fetal \& neonatal medicine. 2013; 26: 339-46.

37. Roos S, Jansson N, Palmberg I, Säljö K, Powell TL, Jansson T. Mammalian target of rapamycin in the human placenta regulates leucine transport and is down-regulated in restricted fetal growth. The Journal of Physiology. 2007; 582: 449-59.

38. Hung TH, Hsieh TT, Wu CP, Li MJ, Yeh YL, Chen SF. Mammalian target of rapamycin signaling is a mechanistic link between increased endoplasmic reticulum stress and autophagy in the placentas of pregnancies complicated by growth restriction. Placenta. 2017; 60: 9-20.

39. Gauster M, Maninger S, Siwetz M, Deutsch A, El-Heliebi A, Kolb-Lenz $\mathrm{D}$, et al. Downregulation of p53 drives autophagy during human trophoblast differentiation. Cellular and molecular life sciences. 2018; 75: 1839-55.

40. Hung TH, Chen SF, Li MJ, Yeh YL, Hsieh TT. Differential effects of concomitant use of vitamins $\mathrm{C}$ and $\mathrm{E}$ on trophoblast apoptosis and autophagy between normoxia and hypoxia-reoxygenation. PloS one. 2010; 5: e12202.

41. Nakashima A, Cheng SB, Ikawa M, Yoshimori T, Huber WJ, Menon R, et al. Evidence for lysosomal biogenesis proteome defect and impaired autophagy in preeclampsia. Autophagy. 2020; 16: 1771-85.

42. Cao B, Macones C, Mysorekar IU. ATG16L1 governs placental infection risk and preterm birth in mice and women. JCI insight. 2016; 1: e86654.

43. Hiyama M, Kusakabe KT, Takeshita A, Sugi S, Kuniyoshi N, Imai H, et al. Nutrient starvation affects expression of LC3 family at the feto-maternal interface during murine placentation. The Journal of veterinary medical science. 2015; 77: 305-11.

44. Chu A, Thamotharan S, Ganguly A, Wadehra M, Pellegrini M, Devaskar SU. Gestational food restriction decreases placental interleukin-10 expression and markers of autophagy and endoplasmic reticulum stress in murine intrauterine growth restriction. Nutrition research. 2016; 36: 1055-67.

45. Zhu HL, Xu XF, Shi XT, Feng YJ, Xiong YW, Nan Y, et al. Activation of autophagy inhibits cadmium-triggered apoptosis in human placental trophoblasts and mouse placenta. Environmental pollution. 2019; 254: 112991.

46. Agrawal V, Jaiswal MK, Mallers T, Katara GK, Gilman-Sachs A, Beaman $\mathrm{KD}$, et al. Altered autophagic flux enhances inflammatory responses during inflammation-induced preterm labor. Scientific reports. 2015; 5: 9410.

47. Suter M, Riek U, Tuerk R, Schlattner U, Wallimann T, Neumann D. Dissecting the role of 5'-AMP for allosteric stimulation, activation, and deactivation of AMP-activated protein kinase. The Journal of biological chemistry. 2006; 281: 32207-16.

48. Jung $\mathrm{CH}$, Ro $\mathrm{SH}$, Cao J, Otto NM, Kim DH. mTOR regulation of autophagy. FEBS letters. 2010; 584: 1287-95.

49. Zhang $\mathrm{Q}, \mathrm{Na} \mathrm{Q}$, Song $\mathrm{W}$. Moderate mammalian target of rapamycin inhibition induces autophagy in HTR8/SVneo cells via O-linked $\beta$-N-acetylglucosamine signaling. The journal of obstetrics and gynaecology research. 2017; 43: 1585-96.

50. Yang HQ, Chandra Y, Zhang ZY. Modulation of Autophagy Through Regulation of 5'-AMP-Activated Protein Kinase Affects Mitophagy and Mitochondrial Function in Primary Human Trophoblasts. Reproductive sciences. 2021; 28:2314-22.

51. Zhang L, Deng X, Shi X, Dong X. Silencing H19 regulated proliferation, invasion, and autophagy in the placenta by targeting miR-18a-5p. Journal of cellular biochemistry. 2019; 120: 9006-15.

52. Mu Q, Wang $\mathrm{L}, \mathrm{Yu} F$, Gao H, Lei $\mathrm{T}$, Li $\mathrm{P}$, et al. Imp2 regulates GBM progression by activating IGF2/PI3K/Akt pathway. Cancer biology \& therapy. 2015; 16: 623-33.

53. Yuan $Y$, Zhao L, Wang X, Lian F, Cai Y. Ligustrazine-induced microRNA-16-5p inhibition alleviates preeclampsia through IGF-2. Reproduction. 2020; 160: 905-17.

54. Riobó NA, Lu K, Ai X, Haines GM, Emerson CP, Jr. Phosphoinositide 3-kinase and Akt are essential for Sonic Hedgehog signaling. Proceedings of the National Academy of Sciences of the United States of America. 2006; 103: 4505-10. 
55. Genbacev O, Zhou Y, Ludlow JW, Fisher SJ. Regulation of human placental development by oxygen tension. Science. 1997; 277: 1669-72.

56. Azad MB, Chen Y, Gibson SB. Regulation of autophagy by reactive oxygen species (ROS): implications for cancer progression and treatment. Antioxidants \& redox signaling. 2009; 11: 777-90.

57. Scherz-Shouval R, Elazar Z. ROS, mitochondria and the regulation of autophagy. Trends in cell biology. 2007; 17: 422-7.

58. Hung TH, Hsieh TT, Chen SF, Li MJ, Yeh YL. Autophagy in the human placenta throughout gestation. PloS one. 2013; 8: e83475.

59. Vangrieken P, Al-Nasiry S, Bast A, Leermakers PA, Tulen CBM, Janssen GMJ, et al. Hypoxia-induced mitochondrial abnormalities in cells of the placenta. PloS one. 2021; 16: e0245155.

60. Li YX, Long DL, Liu J, Qiu D, Wang J, Cheng X, et al. Gestational diabetes mellitus in women increased the risk of neonatal infection via inflammation and autophagy in the placenta. Medicine. 2020; 99: e22152.

61. Yamanaka-Tatematsu M, Nakashima A, Fujita N, Shima T, Yoshimori T, Saito S. Autophagy induced by HIFla overexpression supports trophoblast invasion by supplying cellular energy. PloS one. 2013; 8: e76605.

62. Zhou X, Han TL, Chen H, Baker PN, Qi H, Zhang H. Impaired mitochondrial fusion, autophagy, biogenesis and dysregulated lipid metabolism is associated with preeclampsia. Experimental cell research. 2017; 359: 195-204.

63. Ma Z, Chen C, Tang P, Zhang H, Yue J, Yu Z. BNIP3 induces apoptosis and protective autophagy under hypoxia in esophageal squamous cell carcinoma cell lines: BNIP3 regulates cell death. Diseases of the Esophagus. 2017; 30: 1-8.

64. Marquez RT, Xu L. Bcl-2:Beclin 1 complex: multiple, mechanisms regulating autophagy/apoptosis toggle switch. American journal of cancer research. 2012; 2: 214-21.

65. Kalkat M, Garcia J, Ebrahimi J, Melland-Smith M, Todros T, Post M, et al. Placental autophagy regulation by the BOK-MCL1 rheostat. Autophagy. 2013; 9: 2140-53.

66. Han D, Jiang L, Gu X, Huang S, Pang J, Wu Y, et al. SIRT3 deficiency is resistant to autophagy-dependent ferroptosis by inhibiting the AMPK/mTOR pathway and promoting GPX4 levels. Journal of cellular physiology. 2020; 235: 8839-51.

67. Ji L, Chen Z, Xu Y, Xiong G, Liu R, Wu C, et al. Systematic Characterization of Autophagy in Gestational Diabetes Mellitus. Endocrinology. 2017; 158: 2522-32.

68. Wang $\mathrm{Y}$, Ji L, Peng $\mathrm{Z}$, Lai $\mathrm{R}$, Zhang $\mathrm{X}, \mathrm{Xu} \mathrm{Y}$, et al. Silencing DAPK3 blocks the autophagosome-lysosome fusion by mediating SNAP29 in trophoblast cells under high glucose treatment. Molecular and cellular endocrinology. 2020; 502: 110674.

69. Ji Y, Zhang W, Yang J, Li C. MiR-193b inhibits autophagy and apoptosis by targeting IGFBP5 in high glucose-induced trophoblasts. Placenta. 2020; 101: 185-93.

70. Chen B, Longtine MS, Nelson DM. Hypoxia induces autophagy in primary human trophoblasts. Endocrinology. 2012; 153: 4946-54.

71. Oh SY, Choi SJ, Kim KH, Cho EY, Kim JH, Roh CR. Autophagy-related proteins, LC3 and Beclin-1, in placentas from pregnancies complicated by preeclampsia. Reproductive sciences. 2008; 15: 912-20.

72. Sagrillo-Fagundes L, Bienvenue-Pariseault J, Vaillancourt C. Melatonin: The smart molecule that differentially modulates autophagy in tumor and normal placental cells. PloS one. 2019; 14: e0202458.

73. Li M, Sun $T$, Wu X, An P, Wu X, Dang $H$. Autophagy in the HTR-8/SVneo Cell Oxidative Stress Model Is Associated with the NLRP1 Inflammasome. Oxidative medicine and cellular longevity. 2021; 2021: 2353504.

74. Lee HM, Choi KC. Cigarette smoke extract and isoprene resulted in the induction of apoptosis and autophagy in human placenta choriocarcinoma JEG-3 cells. Environmental toxicology. 2018; 33: 178-90.

75. Cha HH, Hwang JR, Kim HY, Choi SJ, Oh SY, Roh CR. Autophagy induced by tumor necrosis factor a mediates intrinsic apoptosis in trophoblastic cells. Reproductive sciences. 2014; 21: 612-22.

76. Li M, Wu X, An P, Dang H, Liu Y, Liu R. Effects of resveratrol on autophagy and the expression of inflammasomes in a placental trophoblast oxidative stress model. Life sciences. 2020; 256: 117890.

77. Seno K, Tanikawa N, Takahashi H, Ohkuchi A, Suzuki H, Matsubara S, et al. Oxygen concentration modulates cellular senescence and autophagy in human trophoblast cells. American journal of reproductive immunology. 2018; 79: e12826.

78. Aoki A, Nakashima A, Kusabiraki T, Ono Y, Yoshino O, Muto M, et al. Trophoblast-Specific Conditional Atg7 Knockout Mice Develop Gestational Hypertension. The American journal of pathology. 2018; 188: 2474-86.

79. Wang $\mathrm{Y}$, Zhang $\mathrm{H}$, Zhang $\mathrm{Y}, \mathrm{Li} \mathrm{X}, \mathrm{Hu} \mathrm{X}$, Wang $\mathrm{X}$. Decorin promotes apoptosis and autophagy via suppressing c-Met in HTR- 8 trophoblasts. Reproduction. 2020; 159: 669-77.
80. Oh SY, Hwang JR, Choi M, Kim YM, Kim JS, Suh YL, et al. Autophagy regulates trophoblast invasion by targeting NF-KB activity. Scientific reports. 2020; 10: 14033 .

81. Zhou F, Wang Y, Tan Y, Wu C, Chen Y. HMGB1 regulates lipopolysaccharide-induced cellular dysfunction in HTR8/SVneo cells: Implications for the role of HMGB1 in unexplained spontaneous miscarriage. Placenta. 2021; 112: 16-22.

82. Zhang Y, Hu X, Gao G, Wang Y, Chen P, Ye Y. Autophagy protects against oxidized low density lipoprotein-mediated inflammation associated with preeclampsia. Placenta. 2016; 48: 136-43.

83. Liu J, Song G, Meng T, Zhao G. UL16-Binding Protein 1 Induced HTR-8/SVneo Autophagy via NF-kB Suppression Mediated by TNF-a Secreted through uNK Cells. BioMed research international. 2020; 2020: 9280372.

84. Walker OS, Gurm H, Sharma R, Verma N, May LL, Raha S. Delta-9-tetrahydrocannabinol inhibits invasion of HTR8/SVneo human extravillous trophoblast cells and negatively impacts mitochondrial function. Scientific reports. 2021; 11: 4029.

85. Milane L, Trivedi M, Singh A, Talekar M, Amiji M. Mitochondrial biology, targets, and drug delivery. Journal of Controlled Release. 2015; 207: 40-58.

86. Vande Velde C, Cizeau J, Dubik D, Alimonti J, Brown T, Israels S, et al. BNIP3 and genetic control of necrosis-like cell death through the mitochondrial permeability transition pore. Molecular and cellular biology. 2000; 20: 5454-68.

87. Bak DH, Na J, Im SI, Oh CT, Kim JY, Park SK, et al. Antioxidant effect of human placenta hydrolysate against oxidative stress on muscle atrophy. Journal of cellular physiology. 2019; 234: 1643-58.

88. Seok J, Jun S, Cho J, Park S, Lee JO, Kim GJ. Human placenta-derived mesenchymal stem cells induce trophoblast invasion via dynamic effects on mitochondrial function. Journal of cellular physiology. 2021.

89. Choi JH, Lee HJ, Yang TH, Kim GJ. Effects of hypoxia inducible factors-1a on autophagy and invasion of trophoblasts. Clinical and experimental reproductive medicine. 2012; 39: 73-80.

90. van Uden P, Kenneth NS, Rocha S. Regulation of hypoxia-inducible factor-1alpha by NF-kappaB. The Biochemical journal. 2008; 412: 477-84.

91. Armant DR. Autophagy's expanding role in development: implantation is next. Endocrinology. 2011; 152: 1739-41.

92. Ekizceli G, Inan S, Oktem G, Onur E, Ozbilgin K. Assessment of mTOR pathway molecules during implantation in rats. Biotechnic \& histochemistry. 2017; 92: 450-8

93. Chen X, He J, Ding Y, Zeng L, Gao R, Cheng S, et al. The role of MTOR in mouse uterus during embryo implantation. Reproduction. 2009; 138: 351-6.

94. Su Y, Zhang JJ, He JL, Liu XQ, Chen XM, Ding YB, et al. Endometrial autophagy is essential for embryo implantation during early pregnancy. Journal of molecular medicine. 2020; 98: 555-67.

95. Lee JE, Oh HA, Song H, Jun JH, Roh CR, Xie H, et al. Autophagy regulates embryonic survival during delayed implantation. Endocrinology. 2011; 152: 2067-75.

96. Hemberger M, Hanna CW, Dean W. Mechanisms of early placental development in mouse and humans. Nature reviews Genetics. 2020; 21: $27-43$.

97. Chakraborty S, Bose R, Islam S, Das S, Ain R. Harnessing Autophagic Network Is Essential for Trophoblast Stem Cell Differentiation. Stem cells and development. 2020; 29: 682-94.

98. Zhang QX, Na Q, Song W. Altered expression of mTOR and autophagy in term normal human placentas. Romanian journal of morphology and embryology. 2017; 58: 517-26.

99. Dimasuay KG, Gong L, Rosario F, McBryde E, Spelman T, Glazier J, et al. Impaired placental autophagy in placental malaria. PloS one. 2017; 12: e0187291.

100. Yin X, Gao R, Geng Y, Chen X, Liu X, Mu X, et al. Autophagy regulates abnormal placentation induced by folate deficiency in mice. Molecular human reproduction. 2019; 25: 305-19.

101. Huang X, Han X, Huang Z, Yu M, Zhang Y, Fan Y, et al. Maternal pentachlorophenol exposure induces developmental toxicity mediated by autophagy on pregnancy mice. Ecotoxicology and environmental safety. 2019; 169: 829-36.

102. Díaz M, García C, Sebastiani G, de Zegher F, López-Bermejo A, Ibáñez L. Placental and Cord Blood Methylation of Genes Involved in Energy Homeostasis: Association With Fetal Growth and Neonatal Body Composition. Diabetes. 2017; 66: 779-84

103. Arikawa T, Liao S, Shimada H, Inoue T, Sakata-Haga H, Nakamura T, et al. Galectin-4 expression is down-regulated in response to autophagy during differentiation of rat trophoblast cells. Scientific reports. 2016; 6: 32248 . 
104. He MY, Wang G, Han SS, Jin Y, Li H, Wu X, et al. Nrf2 signalling and autophagy are involved in diabetes mellitus-induced defects in the development of mouse placenta. Open biology. 2016; 6: 160064.

105. Hung TH, Chen SF, Lo LM, Li MJ, Yeh YL, Hsieh TT. Increased autophagy in placentas of intrauterine growth-restricted pregnancies. PloS one. 2012; 7: e40957.

106. Feng X, Wei Z, Tao X, Du Y, Wu J, Yu Y, et al. PLAC8 promotes the autophagic activity and improves the growth priority of human trophoblast cells. FASEB journal. 2021; 35: e21351.

107. Turco MY, Moffett A. Development of the human placenta. Development. 2019; 146: dev163428.

108. Ermini L, Farrell A, Alahari S, Ausman J, Park C, Sallais J, et al. Ceramide-Induced Lysosomal Biogenesis and Exocytosis in Early-Onset Preeclampsia Promotes Exosomal Release of SMPD1 Causing Endothelial Dysfunction. Frontiers in cell and developmental biology. 2021; 9: 652651 .

109. Zhao H, Gong L, Wu S, Jing T, Xiao X, Cui Y, et al. The Inhibition of Protein Kinase $C \beta$ Contributes to the Pathogenesis of Preeclampsia by Activating Autophagy. EBioMedicine. 2020; 56: 102813.

110. Nakabayashi Y, Nakashima A, Yoshino O, Shima T, Shiozaki A, Adachi $\mathrm{T}$, et al. Impairment of the accumulation of decidual T cells, NK cells, and monocytes, and the poor vascular remodeling of spiral arteries, were observed in oocyte donation cases, regardless of the presence or absence of preeclampsia. Journal of reproductive immunology. 2016; 114: 65-74.

111. Holland OJ, Hickey AJR, Alvsaker A, Moran S, Hedges C, Chamley LW, et al. Changes in mitochondrial respiration in the human placenta over gestation. Placenta. 2017; 57: 102-12.

112. Ausman J, Abbade J, Ermini L, Farrell A, Tagliaferro A, Post M, et al. Ceramide-induced BOK promotes mitochondrial fission in preeclampsia. Cell death \& disease. 2018; 9: 298.

113. Quinn KE, Ashley AK, Reynolds LP, Grazul-Bilska AT, Ashley RL. Activation of the CXCL12/CXCR4 signaling axis may drive vascularization of the ovine placenta. Domestic animal endocrinology. 2014; 47: 11-21.

114. Runyan CL, McIntosh SZ, Maestas MM, Quinn KE, Boren BP, Ashley RL. CXCR4 signaling at the ovine fetal-maternal interface regulates vascularization, CD34+ cell presence, and autophagy in the endometrium + . Biology of reproduction. 2019; 101: 102-11.

115. Chen X, Liu J, He B, Li Y, Liu S, Wu B, et al. Vascular endothelial growth factor (VEGF) regulation by hypoxia inducible factor-1 alpha (HIF1A) starts and peaks during endometrial breakdown, not repair, in a mouse menstrual-like model. Human reproduction. 2015; 30: 2160-70.

116. Macklon NS, Brosens JJ. The human endometrium as a sensor of embryo quality. Biology of reproduction. 2014; 91: 98

117. Vinketova K, Mourdjeva M, Oreshkova T. Human Decidual Stromal Cells as a Component of the Implantation Niche and a Modulator of Maternal Immunity. Journal of Pregnancy. 2016; 2016: 8689436.

118. Rhee JS, Saben JL, Mayer AL, Schulte MB, Asghar Z, Stephens C, et al. Diet-induced obesity impairs endometrial stromal cell decidualization: a potential role for impaired autophagy. Human reproduction. 2016; 31: 1315-26.

119. Oestreich AK, Chadchan SB, Popli P, Medvedeva A, Rowen MN, Stephens CS, et al. The Autophagy Gene Atg16L1 is Necessary for Endometrial Decidualization. Endocrinology. 2020; 161: bqz039.

120. Mestre Citrinovitz AC, Strowitzki T, Germeyer A. Decreased Autophagy Impairs Decidualization of Human Endometrial Stromal Cells: A Role for ATG Proteins in Endometrial Physiology. International journal of molecular sciences. 2019; 20: 3066.

121. Okada $H$, Tsuzuki $T$, Murata $H$. Decidualization of the human endometrium. Reproductive medicine and biology. 2018; 17: 220-7.

122. Yin X, Pavone ME, Lu Z, Wei J, Kim JJ. Increased activation of the PI3K/AKT pathway compromises decidualization of stromal cells from endometriosis. The Journal of clinical endocrinology and metabolism. 2012; 97: E35-43.

123. Brunet A, Bonni A, Zigmond MJ, Lin MZ, Juo P, Hu LS, et al. Akt promotes cell survival by phosphorylating and inhibiting a Forkhead transcription factor. Cell. 1999; 96: 857-68.

124. Li L, Zviti R, Ha C, Wang ZV, Hill JA, Lin F. Forkhead box O3 (FoxO3) regulates kidney tubular autophagy following urinary tract obstruction. The Journal of biological chemistry. 2017; 292: 13774-83.

125. Audesse AJ, Dhakal S, Hassell L-A, Gardell Z, Nemtsova Y, Webb AE. FOXO3 directly regulates an autophagy network to functionally regulate proteostasis in adult neural stem cells. PLoS genetics. 2019; 15: e1008097.

126. Cho HJ, Baek MO, Khaliq SA, Chon SJ, Son KH, Lee SH, et al. Microgravity inhibits decidualization via decreasing Akt activity and FOXO3a expression in human endometrial stromal cells. Scientific reports. 2019; 9: 12094.

127. Zhang Y, Gao R, Zhang L, Geng Y, Chen Q, Chen X, et al. AMPK/mTOR downregulated autophagy enhances aberrant endometrial decidualization in folate-deficient pregnant mice. Journal of cellular physiology. 2021.

128. Chen Q, Gao R, Geng Y, Chen X, Liu X, Zhang L, et al. Decreased autophagy was implicated in the decreased apoptosis during decidualization in early pregnant mice. Journal of molecular histology. 2018; 49: 589-97.

129. Kurita T, Lee K-j, Saunders PTK, Cooke PS, Taylor JA, Lubahn DB, et al. Regulation of Progesterone Receptors and Decidualization in Uterine Stroma of the Estrogen Receptor-a Knockout Mouse1. Biology of reproduction. 2001; 64: 272-83.

130. Chen W, Lu S, Yang C, Li N, Chen X, He J, et al. Hyperinsulinemia restrains endometrial angiogenesis during decidualization in early pregnancy. The Journal of endocrinology. 2019; 243: 137-48.

131. Mir SU, Schwarze SR, Jin L, Zhang J, Friend W, Miriyala S, et al. Progesterone receptor membrane component 1/Sigma-2 receptor associates with MAP1LC3B and promotes autophagy. Autophagy. 2013; 9: 1566-78.

132. Choi J, Jo M, Lee E, Oh YK, Choi D. The role of autophagy in human endometrium. Biology of reproduction. 2012; 86: 70.

133. Mei J, Zhu XY, Jin LP, Duan ZL, Li DJ, Li MQ. Estrogen promotes the survival of human secretory phase endometrial stromal cells via CXCL12/CXCR4 up-regulation-mediated autophagy inhibition. Human reproduction. 2015; 30: 1677-89.

134. de la Torre P, Fernández-de la Torre M, Flores AI. Premature senescence of placental decidua cells as a possible cause of miscarriage produced by mycophenolic acid. Journal of biomedical science. 2021; 28: 3 .

135. Xiong Q, Li W, Li P, Yang M, Wu C, Eichinger L. The Role of ATG16 in Autophagy and The Ubiquitin Proteasome System. Cells. 2018; 8.

136. Pfefferle A, Jacobs B, Haroun-Izquierdo A, Kveberg L, Sohlberg E, Malmberg KJ. Deciphering Natural Killer Cell Homeostasis. Frontiers in immunology. 2020; 11: 812.

137. Lash GE, Robson SC, Bulmer JN. Review: Functional role of uterine natural killer (uNK) cells in human early pregnancy decidua. Placenta. 2010; 31 Suppl: S87-92.

138. Lanier LL. NK cell receptors. Annual review of immunology. 1998; 16: 359-93.

139. Flórez-Álvarez L, Hernandez JC, Zapata W. NK Cells in HIV-1 Infection: From Basic Science to Vaccine Strategies. Frontiers in immunology. 2018; 9: 2290.

140. Hodgins JJ, Khan ST, Park MM, Auer RC, Ardolino M. Killers 2.0: NK cell therapies at the forefront of cancer control. The Journal of clinical investigation. 2019; 129: 3499-510.

141. Berkkanoglu M, Arici A. Immunology and endometriosis. American journal of reproductive immunology. 2003; 50: 48-59.

142. Carlino C, Stabile H, Morrone S, Bulla R, Soriani A, Agostinis C, et al. Recruitment of circulating NK cells through decidual tissues: a possible mechanism controlling NK cell accumulation in the uterus during early pregnancy. Blood. 2008; 111: 3108-15.

143. Liu Y, Gao S, Zhao Y, Wang H, Pan Q, Shao Q. Decidual Natural Killer Cells: A Good Nanny at the Maternal-Fetal Interface During Early Pregnancy. 2021; 12: 663660.

144. Faas MM, de Vos P. Uterine NK cells and macrophages in pregnancy. Placenta. 2017; 56: 44-52.

145. Xu L, Li Y, Sang Y, Li D-J, Du M. Crosstalk Between Trophoblasts and Decidual Immune Cells: The Cornerstone of Maternal-Fetal Immunotolerance. Frontiers in immunology. 2021; 12: 642392-.

146. Hanna J, Goldman-Wohl D, Hamani Y, Avraham I, Greenfield C, Natanson-Yaron S, et al. Decidual NK cells regulate key developmental processes at the human fetal-maternal interface. Nature medicine. 2006; 12: 1065-74

147. Jabrane-Ferrat N. Features of Human Decidual NK Cells in Healthy Pregnancy and During Viral Infection. Frontiers in Immunology. 2019; 10: 1397.

148. Tan HX, Yang SL, Li MQ, Wang HY. Autophagy suppression of trophoblast cells induces pregnancy loss by activating decidual NK cytotoxicity and inhibiting trophoblast invasion. Cell communication and signaling. 2020; 18: 73 .

149. Liu J, Song G, Lin X, Pang X, Meng T. Upregulated unique long 16 binding protein 1 detected in preeclamptic placenta affects human extravillous trophoblast cell line (HTR-8/SVneo) invasion by modulating the function of uterine natural killer cells. Experimental and therapeutic medicine. 2017; 13: 1447-55.

150. Mei J, Zhou WJ, Zhu XY, Lu H, Wu K, Yang HL, et al. Suppression of autophagy and HCK signaling promotes PTGS2(high) FCGR3(-) NK cell differentiation triggered by ectopic endometrial stromal cells. Autophagy. 2018; 14: 1376-97.

151. Yu JJ, Sun HT, Zhang ZF, Shi RX, Liu LB, Shang WQ, et al. IL15 promotes growth and invasion of endometrial stromal cells and inhibits killing activity of NK cells in endometriosis. Reproduction. 2016; 152: 151-60. 
152. Zhang B, Zhou WJ, Gu CJ, Wu K, Yang HL, Mei J, et al. The ginsenoside PPD exerts anti-endometriosis effects by suppressing estrogen receptor-mediated inhibition of endometrial stromal cell autophagy and NK cell cytotoxicity. Cell death \& disease. 2018; 9: 574.

153. Zhang M, Zhu H, Ding Y, Liu Z, Cai Z, Zou MH. AMP-activated protein kinase a1 promotes atherogenesis by increasing monocyte-to-macrophage differentiation. The Journal of biological chemistry. 2017; 292: 7888-903.

154. Zhang Y, Morgan MJ, Chen K, Choksi S, Liu ZG. Induction of autophagy is essential for monocyte-macrophage differentiation. Blood. 2012; 119: 2895-905.

155. Lindau R, Vondra S, Spreckels J, Solders M, Svensson-Arvelund J, Berg $\mathrm{G}$, et al. Decidual stromal cells support tolerance at the human foetal-maternal interface by inducing regulatory M2 macrophages and regulatory T-cells. Journal of reproductive immunology. 2021; 146: 103330.

156. Kim H, Wang SY, Kwak G, Yang Y, Kwon IC, Kim SH. Exosome-Guided Phenotypic Switch of M1 to M2 Macrophages for Cutaneous Wound Healing. Advanced science. 2019; 6: 1900513.

157. Wu WK, Llewellyn OP, Bates DO, Nicholson LB, Dick AD. IL-10 regulation of macrophage VEGF production is dependent on macrophage polarisation and hypoxia. Immunobiology. 2010; 215: 796-803.

158. Wheeler KC, Jena MK, Pradhan BS, Nayak N, Das S, Hsu CD, et al. VEGF may contribute to macrophage recruitment and M2 polarization in the decidua. PloS one. 2018; 13: e0191040.

159. Mantovani A, Biswas SK, Galdiero MR, Sica A, Locati M. Macrophage plasticity and polarization in tissue repair and remodelling. The Journal of pathology. 2013; 229: 176-85.

160. Xuan W, Qu Q, Zheng B, Xiong S, Fan GH. The chemotaxis of M1 and M2 macrophages is regulated by different chemokines. Journal of leukocyte biology. 2015; 97: 61-9.

161. Jena MK, Nayak N, Chen K, Nayak NR. Role of Macrophages in Pregnancy and Related Complications. Archivum immunologiae et therapiae experimentalis. 2019; 67: 295-309.

162. Zhang J, Chu D, Kawamura T, Tanaka K, He S. GRIM-19 repressed hypoxia-induced invasion and EMT of colorectal cancer by repressing autophagy through inactivation of STAT3/HIF-1a signaling axis. Journal of cellular physiology. 2019; 234: 12800-8.

163. Heikkinen J, Möttönen M, Komi J, Alanen A, Lassila O. Phenotypic characterization of human decidual macrophages. Clinical and experimental immunology. 2003; 131: 498-505.

164. Bates MD, Quenby S, Takakuwa K, Johnson PM, Vince GS. Aberrant cytokine production by peripheral blood mononuclear cells in recurrent pregnancy loss? Human reproduction. 2002; 17: 2439-44.

165. Qaddourah RH, Magdoud K, Saldanha FL, Mahmood N, Mustafa FE, Mahjoub T, et al. IL-10 gene promoter and intron polymorphisms and changes in IL-10 secretion in women with idiopathic recurrent miscarriage. Human reproduction. 2014; 29: 1025-34.

166. Park HJ, Lee SJ, Kim SH, Han J, Bae J, Kim SJ, et al. IL-10 inhibits the starvation induced autophagy in macrophages via class I phosphatidylinositol 3-kinase (PI3K) pathway. Molecular immunology. 2011; 48: 720-7.

167. Maneechotesuwan K, Kasetsinsombat K, Wongkajornsilp A, Barnes PJ. Role of autophagy in regulating interleukin-10 and the responses to corticosteroids and statins in asthma. Clinical and experimental allergy. 2021; 51: 1553-1565

168. Hönig A, Rieger L, Kapp M, Sütterlin M, Dietl J, Kämmerer U. Indoleamine 2,3-dioxygenase (IDO) expression in invasive extravillous trophoblast supports role of the enzyme for materno-fetal tolerance. Journal of reproductive immunology. 2004; 61: 79-86.

169. Cheng H, Huang Y, Huang G, Chen Z, Tang J, Pan L, et al. Effect of the IDO Gene on Pregnancy in Mice with Recurrent Pregnancy Loss. Reproductive sciences. 2021; 28: 52-9.

170. Metz R, Rust S, Duhadaway JB, Mautino MR, Munn DH, Vahanian NN, et al. IDO inhibits a tryptophan sufficiency signal that stimulates mTOR: A novel IDO effector pathway targeted by D-1-methyl-tryptophan. Oncoimmunology. 2012; 1: 1460-8.

171. Yang SL, Tan HX, Niu TT, Liu YK, Gu CJ, Li DJ, et al. The IFN- $\gamma$-IDO1-kynureine pathway-induced autophagy in cervical cancer cell promotes phagocytosis of macrophage. International journal of biological sciences. 2021; 17: 339-52.

172. Chinnadurai R, Copland IB, Ng S, Garcia M, Prasad M, Arafat D, et al. Mesenchymal Stromal Cells Derived From Crohn's Patients Deploy Indoleamine 2,3-dioxygenase-mediated Immune Suppression, Independent of Autophagy. Molecular therapy. 2015; 23: 1248-61.

173. Erlebacher A. Immunology of the maternal-fetal interface. Annual review of immunology. 2013; 31: 387-411.
174. Wang W, Sung N, Gilman-Sachs A, Kwak-Kim J. T Helper (Th) Cell Profiles in Pregnancy and Recurrent Pregnancy Losses: Th1/Th2/Th9/Th17/Th22/Tfh Cells. Frontiers in immunology. 2020; 11: 2025.

175. C NC, Keane J, Lavelle EC, Hope JC, Harris J. Autophagy in the immune response to tuberculosis: clinical perspectives. Clinical and experimental immunology. 2011; 164: 291-300.

176. Lai Y-S, Wahyuningtyas R, Aui S-P, Chang K-T. Autocrine VEGF signalling on M2 macrophages regulates PD-L1 expression for immunomodulation of T cells. 2019; 23: 1257-67.

177. Pompura SL, Dominguez-Villar M. The PI3K/AKT signaling pathway in regulatory T-cell development, stability, and function. Journal of leukocyte biology. 2018: doi: 10.1002/JLB.2MIR0817-349R.

178. Polese B, Gridelet V, Araklioti E, Martens H, Perrier d'Hauterive S, Geenen V. The Endocrine Milieu and CD4 T-Lymphocyte Polarization during Pregnancy. Frontiers in endocrinology. 2014; 5: 106.

179. Saifi B, Rezaee SA, Tajik N, Ahmadpour ME, Ashrafi M, Vakili R, et al. Th17 cells and related cytokines in unexplained recurrent spontaneous miscarriage at the implantation window. Reproductive biomedicine online. 2014; 29: 481-9.

180. Verma P, Nair RR, Budhwar S, Singh V, Bala R, Khanna A, et al. Interleukin-17 gene polymorphisms and the risk of early miscarriage: A case-control study and meta-analysis. Meta Gene. 2018; 17: 206-11.

181. Zhang S, Huang X, Xiu H, Zhang Z, Zhang K, Cai J, et al. The attenuation of Th1 and Th17 responses via autophagy protects against methicillin-resistant Staphylococcus aureus-induced sepsis. Microbes and infection. 2021; 23: 104833.

182. Saito S, Nakashima A, Shima T, Ito M. Th1/Th2/Th17 and Regulatory T-Cell Paradigm in Pregnancy. American Journal of Reproductive Immunology. 2010; 63: 601-10.

183. Wang J, Chen Z, Xiao Z, Weng Y, Yang M, Yang L, et al. Estrogen induces IDO expression via TGF- $\beta$ in chorionic villi and decidua during early stages of pregnancy. International journal of molecular medicine. 2020; 46: 1186-96.

184. Xiang J, Liu X, Ren J, Chen K, Wang HL, Miao YY, et al. How does estrogen work on autophagy? Autophagy. 2019; 15: 197-211.

185. Tan S, Bajalovic N, Wong ESP, Lin VCL. Ligand-activated progesterone receptor $\mathrm{B}$ activates transcription factor $\mathrm{EB}$ to promote autophagy in human breast cancer cells. Experimental cell research. 2019; 382: 111433.

186. Szekeres-Bartho J, Reznikoff-Etievant MF, Varga P, Pichon MF, Varga Z, Chaouat G. Lymphocytic progesterone receptors in normal and pathological human pregnancy. Journal of reproductive immunology. 1989; 16: 239-47.

187. Li MO, Flavell RA. TGF-beta: a master of all T cell trades. Cell. 2008; 134: 392-404.

188. Latifi Z, Nejabati HR, Abroon S, Mihanfar A, Farzadi L, Hakimi P, et al. Dual role of TGF- $\beta$ in early pregnancy: clues from tumor progression. Biology of reproduction. 2019; 100: 1417-30

189. Lafontaine L, Chaudhry P, Lafleur MJ, Van Themsche C, Soares MJ, Asselin E. Transforming growth factor Beta regulates proliferation and invasion of rat placental cell lines. Biology of reproduction. 2011; 84: 553-9.

190. Ni N, Li Q. TGF $\beta$ superfamily signaling and uterine decidualization. Reproductive biology and endocrinology. 2017; 15: 84.

191. Ghavami S, Cunnington RH, Gupta S, Yeganeh B, Filomeno KL, Freed $\mathrm{DH}$, et al. Autophagy is a regulator of TGF- $\beta 1$-induced fibrogenesis in primary human atrial myofibroblasts. Cell death \& disease. 2015; 6: e1696-e.

192. Kiyono K, Suzuki HI, Matsuyama H, Morishita Y, Komuro A, Kano MR, et al. Autophagy is activated by TGF-beta and potentiates TGF-beta-mediated growth inhibition in human hepatocellular carcinoma cells. Cancer research. 2009; 69: 8844-52.

193. Wang WJ, Zhang H, Chen ZQ, Zhang W, Liu XM, Fang JY, et al. Endometrial TGF- $\beta$, IL-10, IL-17 and autophagy are dysregulated in women with recurrent implantation failure with chronic endometritis. Reproductive biology and endocrinology. 2019; 17: 2 .

194. Shigeru S, Akitoshi N. OP0011. Impaired autophagy in extravillous trophoblast may induce poor placentation in preeclampsia. Pregnancy hypertension. 2013; 3: 65-6.

195. He R, Wang M, Zhao C, Shen M, Yu Y, He L, et al. TFEB-driven autophagy potentiates TGF- $\beta$ induced migration in pancreatic cancer cells. Journal of Experimental \& Clinical Cancer Research. 2019; 38: 340.

196. Diejomaoh MF. Recurrent spontaneous miscarriage is still a challenging diagnostic and therapeutic quagmire. Medical principles and practice. 2015; 24 Suppl 1: 38-55.

197. Cai H, Chen L, Zhang M, Xiang W, Su P. Low expression of MFN2 is associated with early unexplained miscarriage by regulating autophagy of trophoblast cells. Placenta. 2018; 70: 34-40. 
198. Jayaram A, Kanninen T, Sisti G, Inglis SR, Morgan N, Witkin SS. Pregnancy History Influences the Level of Autophagy in Peripheral Blood Mononuclear Cells From Pregnant Women. Reproductive sciences. 2018; 25: 1376-81.

199. Yang D, Ding J, Wang Y, Yuan M, Xian S, Zhang L, et al. YY1-PVT1 affects trophoblast invasion and adhesion by regulating mTOR pathway-mediated autophagy. Journal of cellular physiology. 2020; 235: 6637-46.

200. Wei A, Song Y, Ni T, Xiao H, Wan Y, Ren X, et al. Hyperoside attenuates pregnancy loss through activating autophagy and suppressing inflammation in a rat model. Life sciences. 2020; 254: 117735.

201. Zeng X, Liu Q, Yang Y, Jia W, Li S, He D, et al. Placenta-specific protein 8 promotes the proliferation of lung adenocarcinoma PC-9 cells and their tolerance to an epidermal growth factor receptor tyrosine kinase inhibitor by activating the ERK signaling pathway. Oncology letters. 2019; 18: 5621-7.

202. Wu J, Wang X, Shang A, Vella G, Sun Z, Ji P, et al. PLAC8 inhibits oral squamous cell carcinogenesis and epithelial-mesenchymal transition via the Wnt/ $\beta$-catenin and PI3K/Akt/GSK3 $\beta$ signaling pathways. Oncology letters. 2020; 20: 128.

203. Wang Y, Chen B, Longtine MS, Nelson DM. Punicalagin promotes autophagy to protect primary human syncytiotrophoblasts from apoptosis. Reproduction. 2016; 151: 97-104.

204. Mulla MJ, Weel IC, Potter JA, Gysler SM, Salmon JE, Peraçoli MTS, et al. Antiphospholipid Antibodies Inhibit Trophoblast Toll-Like Receptor and Inflammasome Negative Regulators. Arthritis \& rheumatology. 2018; 70: 891-902.

205. Zhou WJ, Chang KK, Wu K, Yang HL, Mei J, Xie F, et al. Rapamycin Synergizes with Cisplatin in Antiendometrial Cancer Activation by Improving IL-27-Stimulated Cytotoxicity of NK Cells. Neoplasia. 2018; 20: 69-79.

206. Lerchbaum E, Obermayer-Pietsch B. Vitamin D and fertility: a systematic review. European journal of endocrinology. 2012; 166: 765-78.

207. Bodnar LM, Catov JM, Simhan HN, Holick MF, Powers RW, Roberts JM. Maternal vitamin D deficiency increases the risk of preeclampsia. The Journal of clinical endocrinology and metabolism. 2007; 92: 3517-22.

208. Mumford SL, Garbose RA, Kim K, Kissell K, Kuhr DL, Omosigho UR, et al. Association of preconception serum 25-hydroxyvitamin D concentrations with livebirth and pregnancy loss: a prospective cohort study. The Lancet Diabetes \& Endocrinology. 2018; 6: 725-32.

209. Hutabarat M, Wibowo N, Obermayer-Pietsch B, Huppertz B. Impact of vitamin $D$ and vitamin $D$ receptor on the trophoblast survival capacity in preeclampsia. PloS one. 2018; 13: e0206725.

210. Tian X, Ma S, Wang Y, Hou L, Shi Y, Yao M, et al. Effects of Placental Ischemia Are Attenuated by 1,25-Dihydroxyvitamin D Treatment and Associated with Reduced Apoptosis and Increased Autophagy. DNA and cell biology. 2016; 35: 59-70.

211. Wilson RL, Buckberry S, Spronk F, Laurence JA, Leemaqz S, O'Leary S, et al. Vitamin D Receptor Gene Ablation in the Conceptus Has Limited Effects on Placental Morphology, Function and Pregnancy Outcome. PloS one. 2015; 10: e0131287.

212. Pi Y, Tian X, Ma J, Zhang H, Huang X. Vitamin D alleviates hypoxia/reoxygenation-induced injury of human trophoblast HTR-8 cells by activating autophagy. Placenta. 2021; 111: 10-8.

213. Du H, Daftary GS, Lalwani SI, Taylor HS. Direct regulation of HOXA10 by $1,25-(\mathrm{OH}) 2 \mathrm{D} 3$ in human myelomonocytic cells and human endometrial stromal cells. Molecular endocrinology. 2005; 19: 2222-33.

214. Rafiee M, Gharagozloo M, Ghahiri A, Mehrabian F, Maracy MR, Kouhpayeh S, et al. Altered Th17/Treg Ratio in Recurrent Miscarriage after Treatment with Paternal Lymphocytes and Vitamin D3: a Double-Blind Placebo-Controlled Study. Iranian journal of immunology. 2015; 12: 252-62. 